\title{
Nano-scale transistors for interfacing with brain: design criteria, progress and prospect
}

\author{
Nazek El-Atab ${ }^{1}$, Sohail F. Shaikh ${ }^{1}$ and Muhammad M. Hussain ${ }^{1,2}$ \\ ${ }^{1}$ MMH Labs, Computer Electrical Mathematical Science and Engineering Division, King Abdullah \\ University of Science and Technology (KAUST), Thuwal 23955-6900, Saudi Arabia. \\ ${ }^{2}$ EECS, University of California, Berkeley, California, USA
}

E-mail: MuhammadMustafa.Hussain@kaust.edu.sa

Received xxxxxx

Accepted for publication $\mathrm{xxxxxx}$

Published xxxxxx

\section{Abstract}

According to the World Health Organization, one quarter of the world population suffers from various neurological disorders ranging from depression to Alzheimer's disease. Thus, understanding the operation mechanism of the brain enables us to help those who are suffering from these diseases. In addition, recent clinical medicine employs electronic brain-implants, despite the fact of being invasive, to treat disorders ranging from severe coronary conditions to traumatic injuries. As a result, the deaf could hear, the blind could see, and the paralyzed could control robotic arms and legs. Due to the requirement of high data management capability with power consumption as low as possible, designing nano-scale transistors as essential I/O electronics is a complex task. Herein, we review the essential design criteria for such nano-scale transistors, progress and prospect for implantable brain-machine-interface (BMI) electronics. This article also discusses their technological challenges for practical implementation.

Keywords: CMOS, organic transistors, sensors, biomedical devices, brain-machine interfacing, brain implantation

\section{Introduction}

The quest for a reliable brain-machine-interface (BMI) that can monitor the activities of all neurons simultaneously at a millisecond resolution poses challenges that, if overcome, could result in a full and deep understanding of the brain behavior and enable future brain-related medical applications. Neurons are the fundamental processing units in the brain and are interconnected via complex networks. Neurons communicate with each other by the opening and closing of ion channels on their membrane, which creates propagating electric currents known as action potentials (AP). APs are either detected by BMIs to analyze the neurons and brain behavior, or generated by BMIs to stimulate neurons and change their activity; a requirement for clinically relevant applications. Therefore, recording and decoding the electrophysiological activities of neurons is at the heart of neuroscience research [1-4].

In 1924, electroencephalography (EEG) was developed by Hans Berger as the first Brain Interface technique and was later used to record brain electrical activity using oscillatory measurements [5]. In 1973, the expression "Brain Computer Interface" (BCI) was first used by Jacques Vidal to explain his project focused on the recording of EEG signals and their computer-based processing [6]. During the following years, several brain-activity recording and mapping techniques have been discovered with steady improvements in spatiotemporal resolution. For example, since 1950s, the number of neurons whose activity can be electrically and simultaneously observed using wired electrodes has doubled every 7 years (mimicking a slower version of Moore's law), currently reaching hundreds of neurons with a temporal resolution of sub-milliseconds [7]. 
Moreover, in addition to electrical based recordings, the developed monitoring techniques have diversified, leading to magnetic resonance, optical and molecular based monitoring (Figure 1), each having its own advantages and disadvantages. For instance, photodetectors have been used to detect the optical signals generated by neurons augmented with fluorescent indicators, however, optical based detection shows difficulty in recording deep brain activity and in achieving high resolution in both temporal and spatial domains. In fact, current optical approaches such as lightsheet illumination can detect $\sim 100,000$ neurons at a $1.25 \mathrm{~s}$ resolution in larval zebra fish [8] while 1-photon fiber scope can detect thousands of neurons at a $\sim 100 \mathrm{~ms}$ resolution in mice [9].

Magnetic resonance imaging (MRI) technique has been used to observe whole brain recordings at $1 \mathrm{~s}$ time resolution based on the radio frequency emissions from excited nuclear spins in a non-invasive manner. However, MRI spatial resolution is too low and thus can not be used to analyze neuron networks in part due to the use of hemodynamic contrast. Alternative techniques based on recording neural activity into biopolymers have been proposed [10-13], however, no demonstrations on neurons have been shown yet.

Till now, intracortical penetrating implants are considered as the BMIs with the highest spatial and temporal resolution for brain activity mapping because they are the only BMIs to enable in vivo single neuron activity monitoring. Intracranial implants are based on microelectrodes with multiple microelectronic sensors (a.k.a microelectrodes arrays (MEA)), and are usually made of rigid material such as silicon to allow the penetration through the pia-matter and the cortex. Examples of such implants include the commercially available Michigan probes, the Utah array (Figure 1(A-B)) and the Hybrid system designed by Wise et al. (Figure 1(C)) [14]. Paradromics startup is also working on the development of a MEA called Neural Input-Output Bus (NIOB) that will use groups of microwire electrodes to record neural activity. Paradromics suggests that it is possible to record and stimulate 1 million neurons using 200,000 microwires (Figure 1(D)).

However, the volume and rigidity of such MEA intracranial implants are resulting in severe damages to the nearby tissues. Latest technologies focus on flexible electronic devices to reduce the rigidity mismatch between tissues and implantable devices while ensuring the penetration of the devices into the tissues. In addition, transistor-based sensors emerged recently due to their capability to reduce the size of the sensors well below MEAs at the micro or nanoscale graphene [15-22].

This review is prepared to emphasize latest advances in nanoscale transistors enabled BMIs with semiconductor nanostructructures, including silicon nanowires (SiNWs),

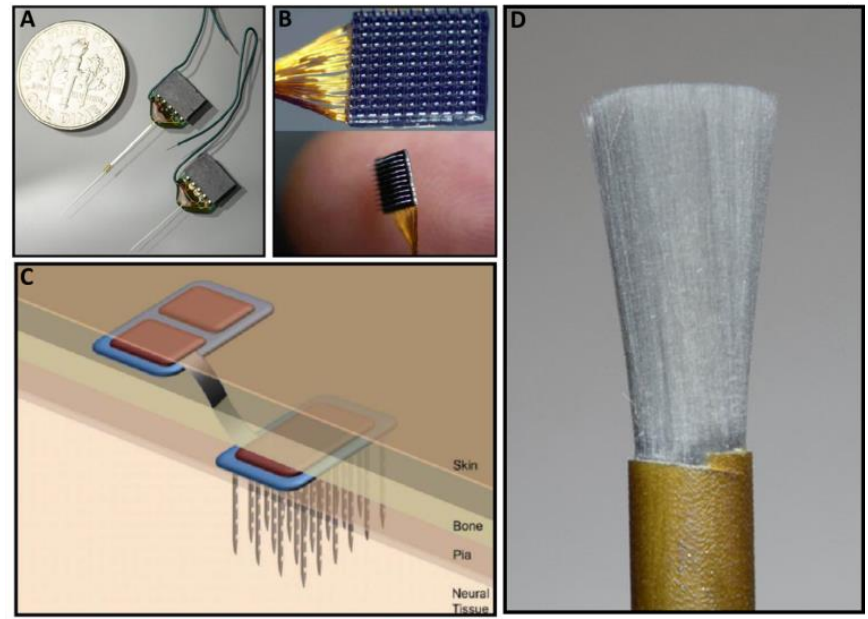

Figure 1. (A) Michigan probes consists of silicon dioxide covered silicon needle with an array of probes and metallic sensors to monitor the neurons activity (B) Utah probes (C) Blackrock Microsystems) based on 100 silicon needles with uninsulated tip for the recordings (C) Hybrid system combining between the Michigan and Utah probes. This system is based on the 3D assembly of Michigan needles on the same implant, however, this results in a higher total volume of the implant suggesting lower bioacceptancy. Reprinted with permission from [14]. (D) Paradromics (C) microwire array bundle connected to CMOS amplifiers.

carbon nanotubes (CNTs), and graphene. First, the requirements for future BMIs in terms of spatio-temporal resolution, power dissipation, volume displacement and biocompatibility are explained. Next, the nano-transistors with different nanomaterials as neuron activity monitoring devices are presented. Multiple probe delivery techniques are then presented including syringe-injectable mesh and macroporous probe methods. Finally, the integration challenges into a complete tetherless BMI system with are discussed.

\section{Design Criteria}

\subsection{Spatio-temporal resolution}

A neuronal spike lasts for $\sim 2 \mathrm{~ms}$ while the rate of neuronal spiking (action potential) varies largely based on the region in the brain and type of neuron. For instance, half of the neurons in the brain are based on cerebellar granule cells and have firing rates of $0.5 \mathrm{~Hz}$ [23-26].

In neocortex, the firing rate is estimated to be around $\sim 0.16$ spikes per second per neuron [27]. In addition, some neurons maybe silent, which means they spike less than once every 10 s [28-29]. However, it has been discussed that sampling all types of neurons at a minimum sampling rate of $1 \mathrm{kHz}$ is essential for several reasons. First, several neural network functions such as spike-timing dependent plasticity rely on temporally precise spiking patterns and therefore require the sampling with milliseconds precision [30-33]. In 
addition, the function of neurons can be highly correlated across large networks, which suggests that neural activity sensors might be subject to high firing rates due to the simultaneous action potentials in the neurons [34].

As a result, a sampling rate of $1 \mathrm{kHz}$ is essential to capture the fast and simultaneous spikes with a single spike resolution, which converts into $7.5 \times 10^{10}$ bits per second to monitor 1 bit per neuron in a mouse brain [35].

Moreover, when a single electrode is used to record the activity of multiple neurons, then a sampling rate of $40 \mathrm{kHz}$ would be necessary to discriminate the different shapes of the spike signals above the noise. Finally, it is possible to compress neural signals before transmission using 1 bit/neuron/ms as the minimal whole brain data rate [35].

\subsection{Power dissipation}

Tissues in the brain can sustain a rise in the local temperature by $2{ }^{\circ} \mathrm{C}$ for hours without getting severely damaged [36]. In $0.5 \mathrm{~g}$ of brain mouse, this rise in temperature corresponds to a power dissipation of $40 \mathrm{~mW}$. This suggests that the neuronal activity monitoring technique should not dissipate more than $40 \mathrm{~mW}$ at steady state in a mouse brain. It should be noted that this value might be under-estimating the actual power dissipation limit since it does not take into consideration other factors such as radiative heat loss, cooling in the cerebrospinal ventricles [37] and conductive heat loss from the brain [38-39]. Moreover, higher power dissipation techniques could be possible to use, however, their experimental time would have to be limited to few minutes instead of several hours [40]. Finally, there is also a limit on the power density of the radiation exposed to human tissue: $\sim 10 \mathrm{~mW} / \mathrm{cm}^{2}$ for radiofrequency electromagnetic radiation $[41], \sim 720 \mathrm{~mW} / \mathrm{cm}^{2}$ for ultrasound radiation, while $\sim 10-100 \mathrm{~mW} / \mathrm{cm}^{2}$ for visible and near infrared exposures up to $1 \mathrm{~ms}$ exposures [42]. Therefore, the exposure of the tissues to heat should be taken into consideration when designing the electronic sensors since the heat can cause a change in the neuronal activity patterns.

\subsection{Volume displacement and biocompatibility}

The insertion of recording probes into the brain causes volume displacement that can potentially disrupt the normal brain functions. It has been suggested that the volume displacement should not be above $1 \%$ of the brain's volume [35]. In fact, previous demonstrations have shown the capability to insert large number of probes in different areas of the brain without compromising its functions. For instance, almost $\sim 100$ electrodes with diameters of $50 \mu \mathrm{m}$ we inserted into different regions of a rat's brain [43] causing
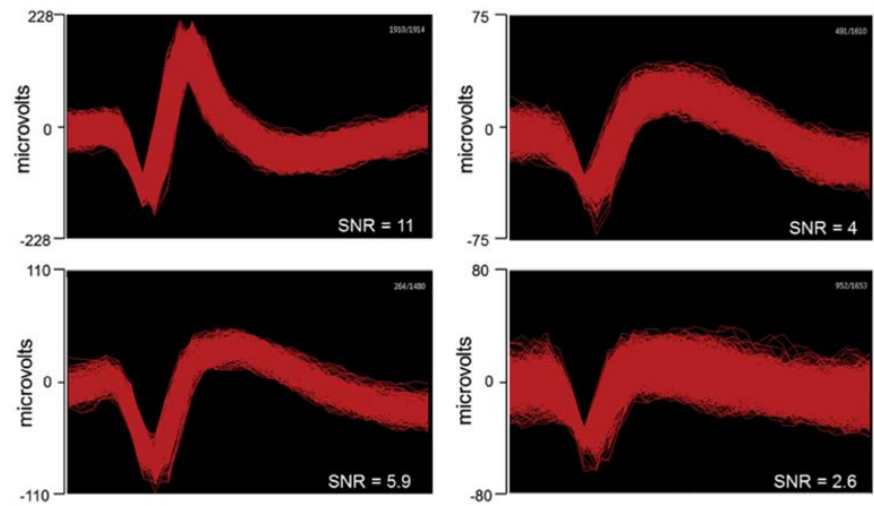

Figure 2. Measured neurons spikes using intracranial Utah probes. The upper left signal shows a SNR of 11 while the bottom right signal shows a 2.6 SNR. Reprinted with permission from [48].

a volume displacement below $0.1 \%$. In addition, almost $\sim 700$ electrodes with diameters of $50 \mu \mathrm{m}$ were implanted in the cortex of a rhesus macaque with a depth of $2.5 \mathrm{~mm}$ [443] causing a volume displacement of less than $0.01 \%$.

Another important factor to consider is the nature/region of the brain volume to be displaced. For example, probes that can cut connectivity in the brain are expected to cause brain damage no matter how small the volume displacement is. On the other hand, a higher volume displacement might be possible if the probes are inserted gradually so that the brain adapts to it without disturbing the brain functions.

Also, it is important that the inserted devices do not affect the blood circulation. In fact, it has been shown that the implant of high density of materials in the brain can cause severe vascular damage resulting in a stroke. High throughput histology has been used recently to define the complete vascular network in a mouse cortex. Such technique can be used to determine the location of key vascular pathways and avoid disrupting their function [45].

Finally, it is crucial that the implanted system is bioacceptable otherwise glial scars will develop. A glial scar is caused by the invasiveness of the inserted probe and results in an inflammatory reaction where glial cells accumulate around the implant device creating a $50 \mu \mathrm{m}$-thick barrier between the probes and the neurons. As a result, measurements with a low signal-to-noise ratio will be initially observed (Figure 2), and as the glial scar thickens in time, a failure of signal detection would occur after a few weeks [46-48]. For instance, the Utah probes insertion allows the measurements up to 18 months while Michigan probes enables neuronal recordings up to 4 months before glial cells start accumulating around the probes. It is worth to mention that the glial scar effect stops when the implant is removed [49].

\section{Nanoscale Transistors in Brain-Machine-Interfaces}

As previously mentioned, transistors have been used recently as biosensors instead of MEAs due to their 
capability to further reduce the size of sensors at the nanoscale. In fact, nano-bioelectonics enable multiplexed, enduring and deep brain recordings of neural activity with high spatial and temporal resolutions [50-53]. The key element in the nano-bioelectronic transistor-based sensors is the nanostructure and nanomaterial used to sense and detect or stimulate the cellular activity around it. Nanostructures show several advantages in bioelectronics applications over larger scale structures. First, nanostructures have a high surface-to-volume ratio which results in a high sensitivity, as a consequence, a small number of external molecules/analytes would be needed to create a detectable electrical signal in the transistor. Another advantage of nanostructures is their small size which is of the same order of magnitude of the biological building blocks, as a result, this enables their seamless integration with cells and tissues and provides unique opportunities for biomedical applications [54-67].

The bioelectrical activity of neurons is based on ionic current flow through the membrane, which changes the electric potential inside and outside the neuron. In order to use the transistor as a biosensor of neuronal activity, a constant voltage is applied and maintained between the source and drain. When a cell is close to the gate oxide surface of the transistor, the extracellular activity modulates the charge carrier's density in the channel of the transistor and therefore causes a variation in the current flowing through the transistor. Thus, the measurements of current variations in the FET allows the detection of the cellular activity around the transistor. The current also depends on the distance and adhesion of the cell on the transistor surface.

\subsection{Silicon nanowires based transistors}

Nanowires are two-dimensional nanomaterials where electrons movement is confined in one-dimension [68-71]. This one-dimensionality of nanowires is the key feature that enables nanowires-based field effect transistors (FETs) to overcome the limitations of planar FETs in terms of sensitivity. As a result, when the analytes bind to the nanowire-based FETs, the measured change in conductance (due to accumulation or depletion of carriers) is more substantial that in the case of planar FETs.

Nanowires based FETs have emerged as promising devices for bioelectronic applications and in specific in BMIs due to their minimal invasiveness. In addition, the contact length along the axon or dendrite of the neuron (transmitting and receving compartments in a neuron, respectively) crossing a nanowire is about $20 \mathrm{~nm}$ which is substantially smaller than in the case of planar FETs or microelectrode arrays. This small size provides several advantages including a better spatial resolution of signal detection without having to take into consideration the error coming from extracellular potentials which can vary over the different portions of a neuron. Another advantage is the capability to integrate the axon's elecments with the dendrite prjections from a single cell. Finally, it is possible to stimulate neuronal activity through the nanowire/axon junction due to the small size of the nanowire. In fact, it has been shown that by applying biphasic pulses to the nanowre/axon junctions and therefore stimulating neuronal activity, somatic spikes have been generated and recorded using intra-cellular elctrodes [54]. In addition, fabricating dense nanowire based FETs is currently achievable which enables the interfacing of arrays of nanowires to inputs and outputs of neurons. As a result, this allows the nanowires array to stimulate, inhibit or reversibly block the propagation of signals through specific pathways throughout the neural network.

In 2006, Lieber et al. first demonstrated the application of open gate $\mathrm{Si}$ nanowire FETs in monitoring extracellular activity from cultured hippocampal neurons. Si nanowire FETs were fabricated using a bottom-up approach where the surface of polylysine was patterned to guide the growh of well-aligned neurons on nanowires, this method resulted in a high yield of nanowires FETs interfacing neurons [17]. Finally, the metal contacts were passivated to avoid their corrosion and failure during harsh conditions of cells culture (Figure 3 (A)). In this work, the authors recorded extracellular activity using the nanowire FETs and the APs using a glass micropipette. Both recordings shows consistent and time correlated results which confirm the reliable measurements of the extracellular activity using nanowire FETs. The fabrication technique allowed for well aligned nanowires on the axon or dendrites compartments of a neuron which enabled direct single neuron activity recordings (Figures $3(\mathrm{~B}-\mathrm{F})$ ). It is worth to mention that these measurements would have been difficult to achieve otherwise due to the larger size of other devices.

Moreover, the authors showed a guided growth of neurons on a rectangular array of nanowire FETs, direct recordings of AP signals was only possible from nanowire FETs which are in contact with the axon or dendrite of the neuron (NW 1, NW2 and NW3); the rest of the FETs (NW4) which are not connected to the neuron could not measure any activity (Figures 3 (B-C), $3(\mathrm{E})$ ). In addition, the array of nanowire FETs in contact with differents site on the neuron is used to study the propagation rate of signals in axon and dendrite; the propagation rates were found to be in the roder of 0.43 $\mathrm{m} / \mathrm{s}$ and $0.16 \mathrm{~m} / \mathrm{s}$, respectively (Figures $4(\mathrm{G}-\mathrm{H})$ ). Finally, Lieber et al. confirmed the potential of nanowire FETs to record multiplexed signals from neurons with subcellular resolution by directing the growth of the axon of a single neuron on the channel of 50 independently addressable FETs (Figures 3 (I-J)). 

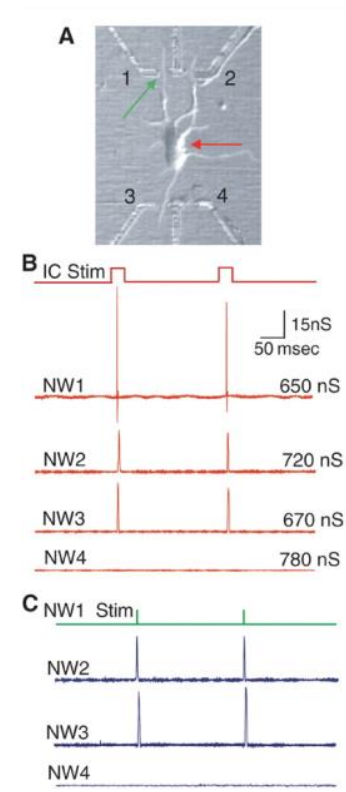

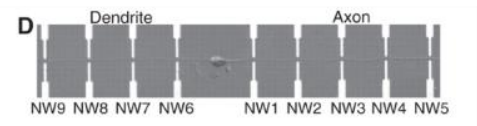

E
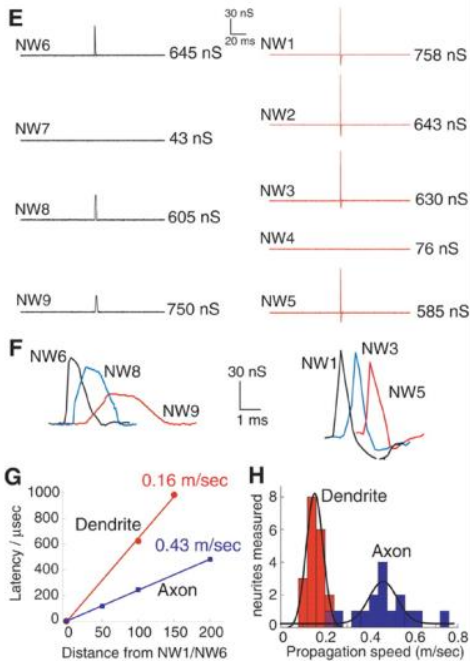

NW4
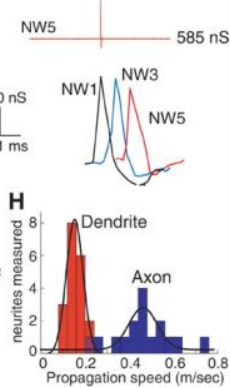

I

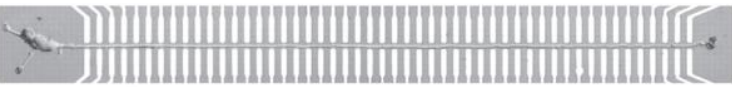

J

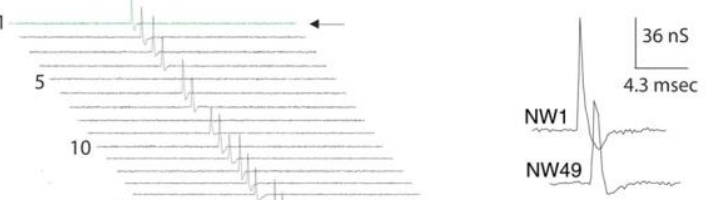

15

20

25
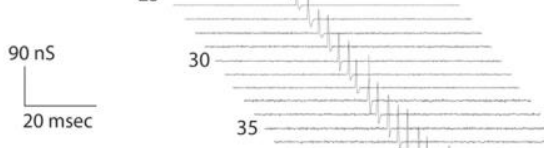

40

45

Figure 3. (A) optical image of a neuron interfaced to three out of the four nanowire FETs array. (B) Traces of nural current stimulations and corresponding nanowire electrical responses (NW). NW4 is not connected to any region of the neuron. (C) Trace of stimulated pulses on NW1 and the response is measured at the nanowire-dendrite junction of NW2 and NW3 (D) Optical image of a neuron showing the dendrite and axon aligned in opposite directions (E) Electrical responses recorded at the NW-axon junctions (right) and NW-dendrite junctions (left) afterintracellular stimulation with a 0.5-nA current pulse for 15-ms, (F) Expansion of peaks from (E) showing the change in peak shape as it propagates along each process. (G) Latency time measured as a function of distance from NW1 and NW6 for axons and dendrites $(\mathrm{H})$ Measured propagation speed through axons and dendrites (I) Optical image of an axon crossing an array of $50 \mathrm{NW}$ devices (J) Measured electrical data from the 50-device array in (I). Reprinted with permission from [17].

In another work, Quing et al. fabricated an array $\mathrm{Si}$ nanowires based FETs on transparent quartz substrates for application in BMI (Figure 4 (A)) [58]. The silicon nanowires of 30 -nm-size in diameter were grown using chemical vapor deposition with gold nanocluster catalyzer. The FETs were next fabricated by aligning the nanowires on the quartz susbtrate followed by electron-beam lithography and deposition of metal contacts $(\mathrm{Ti} / \mathrm{Pd} / \mathrm{Ti})$. Passivation of the contacts was achieved using Si3N4 and SU-8 polymer. The nanowires array was then interfaced with slices of rats brain and resulted in less than $10 \mu \mathrm{m}$ and sub-milliseconds spatio-temporal resolutions. The transparency of the susbtrate emabled the imaging and detection of healthy areas on the upper and lower neuron surfaces. Moreover, the ultrasmall area of the device $\left(0.06 \mu \mathrm{m}^{2}\right)$ with a $3 \mu \mathrm{m}$ array spacing allowedthe integration of highly multiplexed detectors for neuronal activities recordings. This resulted in recordings related to signal propagation in the lateral olfactory tract in addition to neuronal connectiity in the olfactory cortex. Figure 4 (A) shows a schematic of the fabricated nanowire-based FET array on the transparent susbtrate. Figure 4 (B) depicts the nanowire FET array under the brain slice that was stimulated at multiple locations. The propagation rate in the lateral olfactory tract was estimated to be around $2 \mathrm{~m} / \mathrm{s}$ based on the difference between the time and distance of multiple stimulations (Figure 4 (C)). To demonstrate the capability of the nanowire FETs to simultaneously probe and stimulate neuronal activities, a four-by-four array was used to monitor the neurons activities while eight other nanowire FETs were used to stimulate neuronal activities in the lateral olfactory tract (Figure 4 (D)). After activating all neuronal axons using a strong stimulation, the eight nanowire FETs showed similar rescordings irrespectiove of the position of the stimulation (Figure 4 (E)).

However, by sending weaker stimulations, a limited number of axons were activated and as a result, revealing features which are specific to the device (Figure $4(\mathrm{~F})$ ). In fact, by applying weak stimulations, a 2D activity map of all the devices is constructed showing heterogeneous activity and activity correlations with different stimulations spots (Figure $4(\mathrm{G})$ ). For instance, recorded signals from devices 1 and 8 showed clear correlation with stimulation spots on 5 and 8 , whule signals from devices 3 and 4 showed correlation with stimulation spots number 3 and 8 . This proves that the nanowire FETs provide highly localized and multiplexed detection of signals. In fact, this study provided the first demonstration of how localized electrical measurements from neurons using nanowire FETs could help us in visualizing the dynamic neural networks and therefore, allow us to understand circuits and plasticity.

Thus, Si NWFETs have numerous features that make them advantageous for neural activity recording in acute 
brain slices. First of all, previous studies have proven that $\mathrm{Si}$ NWFETs show an excellent sensitivity in the detection of charged biomolecules and single particles. Second, the fabrication of the NWFETs is based on a bottom-up approach which reduces the device to cell separation and promotes an improved nanowire-cell interaction. This results in a higher signal-to-noise ratio in the neural recordings compared to planar FETs. Moreover, the bottom-up approach enables the fabrication of the NWFETs on transparent and flexible substrates which allows obtaining high-resolution optical images of the biological tissue samples. Additionally, the NWFETs typically have an active junction area of around $0.06 \mu \mathrm{m}^{2}$ which is very localized, leading to higher spatial resolution of neural activity recordings compared to planar FETs or MEA with active areas that are larger by a factor up to $10^{4}$. The NWFET based sensors have also shown a high temporal resolution due to their fast intrinsic response.

However, the growth of the bottom-up silicon nanowires requires a catalyst which is generally based on gold nanoparticles, as a result the cost of the overall system increases. Therefore, developing new low-cost catalytic materials for vertical nanowire growth is crucial.

\subsection{Carbon-nanotube based transistors}

In addition to silicon nanowire FETs, single wall carbon nanotubes (SWCNT) have been demonstrated as a biosensor in BMIs due to their high biocompatibility [72] and their capablity to detect $\mathrm{CgA}$ proteins that are released from living neurons. In fact, $\mathrm{CgA}$ is known to have several important biofunctions including fighting against bacterial infection. It has also been confirmed that the appearance of $\mathrm{CgA}$ molecules in plasma is an indicator that can be used to diagnose different diseases such as Parkinson's and Alzheimer's diseases [73-77]. Thus, the detection of $\mathrm{CgA}$ can be used as a detector of specific synaptic activity and as a diagnostic tool for clinical examination. The SWCNT FET is fabriated by first patterning the outer electrodes using photolithography. Next, the 2-nm SWCNTs were transferred to a $\mathrm{SiO}_{2} / \mathrm{Si}$ ubstrate by dop coating it in a solution with $0.125 \%$ CNTs and sodium dodecylbenzenesulfonate (NaDDBS) surfactant [78]. The srufactant is later removed by baking the sample at $200{ }^{\circ} \mathrm{C}$ for 12 hours. Finally. The CNTs are contacted with $\mathrm{Cr}$ followed by a layer of $\mathrm{SiO}_{2}$ for passivation in order to eliminate any electric leakage to the solution. The SWCNTs were then functionalized by
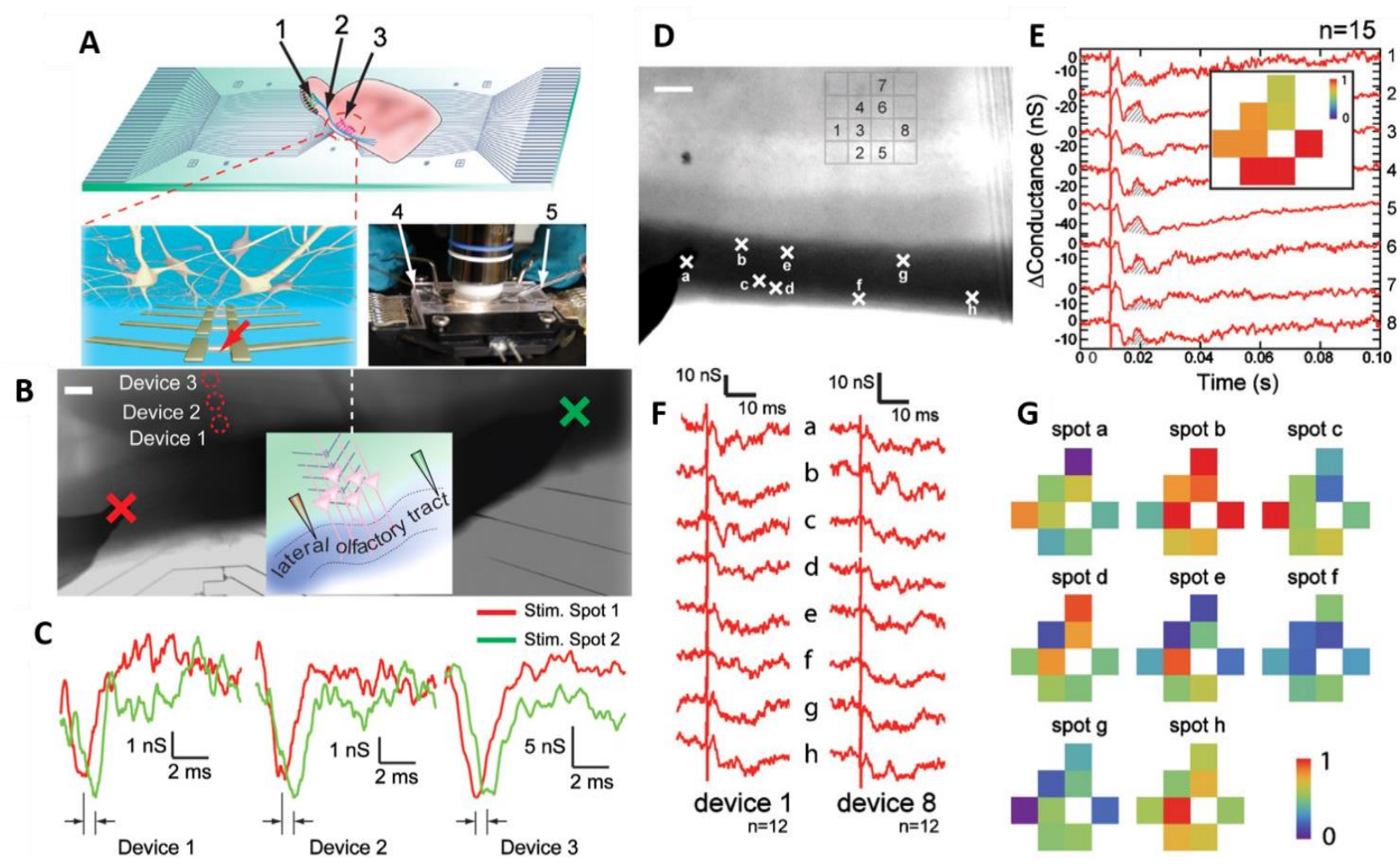

Figure 4. (A) Schematic showing the fabricated silicon nanowire based FET. (B) Nanowire FET array under the brain slice that was stimulated at multiple locations. (C) NWFET conductance measurments when stimulating at two different spots. (D) Optical image of the brain slice over a $4 \times 4$ NWFET array. Signals were detected from the eight NWFETs indicated on the image. Scale bar represents $100 \mu \mathrm{m}$. (E) Signals measured for NWFETs $1-8$ when stimulated with a $400 \mu \mathrm{A}$ pulse for $200 \mu$ s. Inset: normalized map of the recorded signal intensity from the 8 NWFETs. (F) Signals detected by NWFETs 1 and 8 when stimulating at spots A-H with $100 \mu \mathrm{A}$ pulses at $200 \mu \mathrm{s}$. (G) Maps of the relative recorded signal intensity or activity for NWFETs 1-8. Copyright 2009 National Academy of Sciences [58]. 
known antibody for CgA (Figure 5 (A)). The immobilization of the CNTs with $\mathrm{CgA}-\mathrm{Ab}$ was confirmed both electrically and using fluorescent imaging (Figure 5 (B$\mathrm{C})$ ). When the $\mathrm{CgA}$ molecules were brought in contact with the CNT FETs, the threshold voltage is found to shift to negative values indicating the transport of electrons from $\mathrm{CgA}-\mathrm{Ab}$ to the CNTs. In addition, the current output is reduced due to the scattering effect reducing the mobility of the channel carriers (Figure 5 (B)). To confirm the aplicability of $\mathrm{CgA}-\mathrm{Ab}$ in medical applications, it was immersed in fetal bovine serum (FBS) which contains several proteins and small molecules which makes the detection of $\mathrm{CgA}-\mathrm{Ab}$ even more stringent. Figure 5 (D) shows an increase in the current even when $1 \mathrm{nM}$ of $\mathrm{CgA}-\mathrm{Ab}$ was added. Thus, the capability of SWCNTs to selectively detect such small concentration of $\mathrm{CgA}$ in a complex solution makes it suitable for applications for diagnosing neuronal and neuroendocrine cancer [79]. Finally, the authors demonstrated the capability of the fabricated SWCNTs FETs to detect the CgA molecules that were released by the cortical neurons from rats (Figure $5(\mathrm{E})$ ). Glutamate, which is a very known neurotransmiter, is used to stimulate the neurons to release $\mathrm{CgA}$. Figure $5(\mathrm{~F})$ shows an increase in the output current is observed when the $\mathrm{CgA}$ was detected by the CgA-Ab/SWCNT-FETs. The results show the portential of SWCNT-FETs in neuro-medical diagnosis in addition to monitoring the activity of single neurons.
Therefore, in addition to their high biocompatibility for neuron cells, their selectivity, sensitivity, and real-time detection capabilities, CNT based FETs have shown a high sensitivity to proteins released from released from the synaptic terminal of neurons as mediator for neuron inflammation and as markors for the diagnosis of neuroendocrine tumors and neurodegenerative diseases. The disadvantage of CNT based FETs lies in the fabrication process where the growth of CNTs is not conducted directly on the substrate, instead the CNTs are grown seperately and are subsequently coated on the substrate using dip coating technique. This method generally results in multiple CNTs between the two sensing electrodes which affects the resoltuion of the device and its capability to detect single neuron activity. Therefore, it is crucial to improve the fabrication process to enable the probing and reading from single CNT FETs for detection and stimulation of neural activity at the level of individual axons and/or dendrites.

\subsection{Graphene based transistors}

Graphene has also attractive mechanical and electrical properties that make it suitable for neural probes applications [80-84]. However, as in the case of metal electrodes, the minimum detectable feature in graphene FETs is inversely proportional to the square root of the active graphene area, thus a large area would be required to achieve high sensitivity and high spatial resolution [85-89].
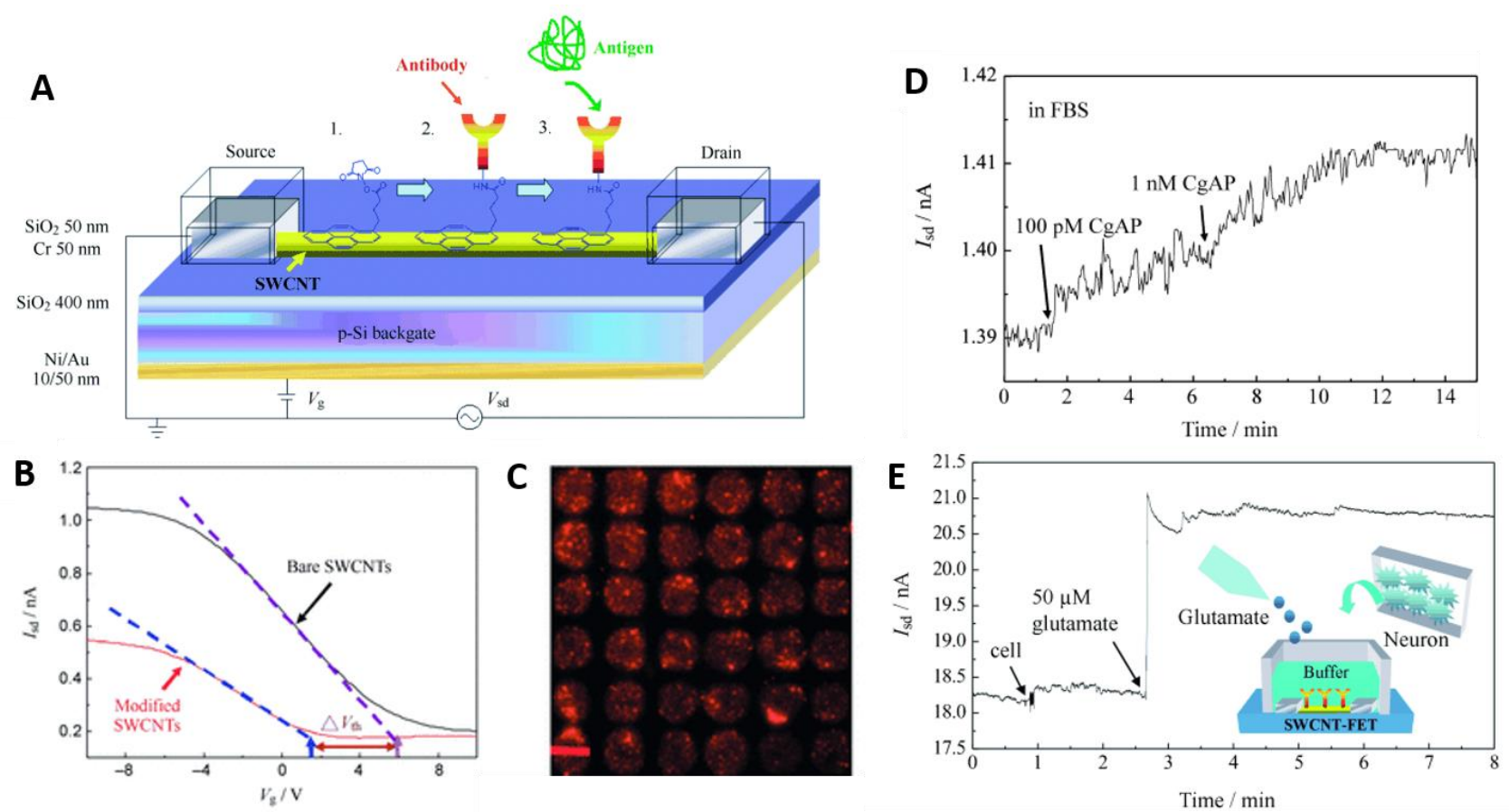

Figure 5. (A) Schematic illustration of a SWCNT-FET sensor (B) Measured Isd-Vg curves before and after the immobilization of CgA$\mathrm{Ab}$ on a SWCNT-FET (C) Fluorescence image of the SWCNTs modified with CgA. Scale bar: $30 \mu \mathrm{m}$. (D) Electric responses of a CgA$\mathrm{Ab} / \mathrm{SWCNT}$-FET to different concentrations of CgA dissolved in FBS. Current was measured at Vsd=10 mV with a modulation frequency of $17.7 \mathrm{~Hz}$. (E) In situ detection of the Isd changes elicited by glutamate at Vsd=10 $\mathrm{mV}$ with a modulation frequency of 377.7 Hz. Reprinted with permission from [78]. 
A

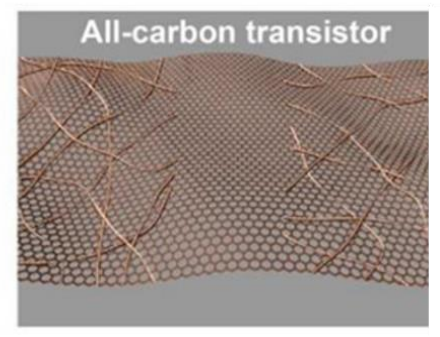

B

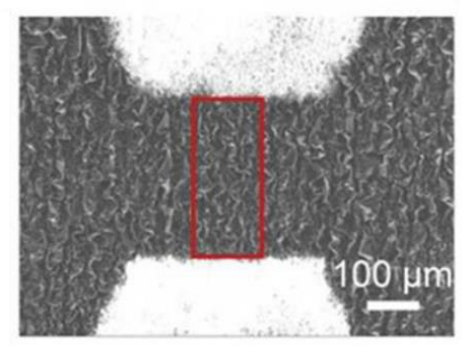

D

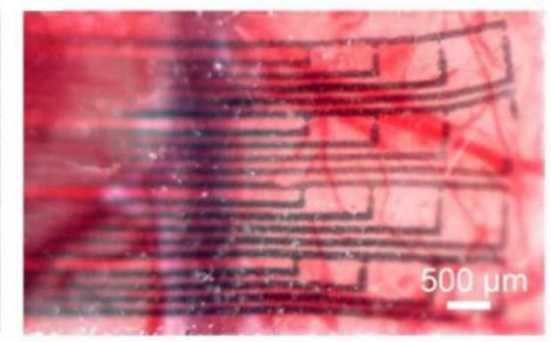

c

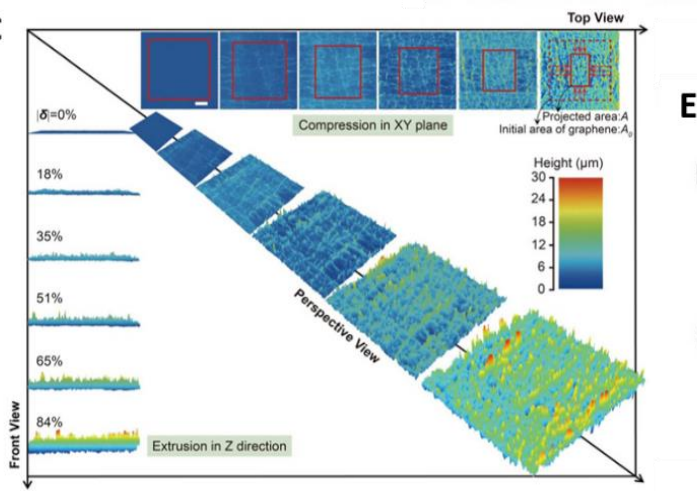

E

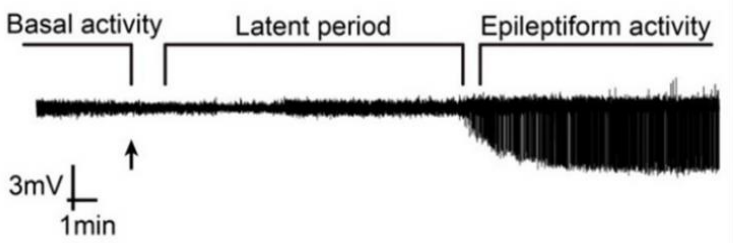

Figure 6. (A) Schematic illustration of a SWCNT-FET sensor (B) Measured Isd-Vg curves before and after the immobilization of CgA$\mathrm{Ab}$ on a SWCNT-FET (C) Fluorescence image of the SWCNTs modified with CgA. Scale bar: $30 \mu \mathrm{m}$. (D) Electric responses of a CgA$\mathrm{Ab} / \mathrm{SWCNT}$-FET to different concentrations of CgA dissolved in FBS. Current was measured at Vsd=10 mV with a modulation frequency of $17.7 \mathrm{~Hz}$. (E) In situ detection of the Isd changes elicited by glutamate at Vsd=10 mV with a modulation frequency of 377.7 Hz. Reprinted with permission from [90]. Copyright 2017 American Chemical Society.

In a recent work led by Yang L. et al., the problem was addressed by compressing the graphene down to $16 \%$ of its initial area and using it in FETs [91]. Regarding the contacts material, CNTs have been used because they show structural inegrity, high flexibility and stable electrical properties over large mechanical deformation while gold, for example, cracks at tensile strains below 1\% [91-98]. The FET is fabricated by first patterning a thin CNT film into source and drain electrodes and then transferring it onto a copper substrate for chemical vapor deposition of graphene. During the growth, metallic graphene is obtained on the CNTs while semiconducting graphene is obtained as the channel of the FET. This resulted in a seamless integration of an all-carbon FET (Figure $6(\mathrm{~A})$ ). Next, the FET was transferred into a biaxially prestrained elastomer, which, upon release, resulted in crumpling the graphene area down to $16 \%$ of its original area (Figure $6(\mathrm{~B})$ ). The resulting graphene has a smaller foot print area but with a larger height which means that the $2 \mathrm{D}$ graphene area was transformed using this technique into a 3D crumpled graphene sheet with high density of high aspect-ratio ridges and vertices as shown in the SEM images. Three dimensional confocal imaging was used to show that the morphology of graphene before and after crumpling did not change and that no cracks were detected (Figure 6 (C)). Next, the graphene based FETs were used as electrocorticography (ECoG ) probes for in vivo recording of brain activity in rats. The FETs were injected into the rat's brain through a craniotomy to access the left cortex of the brain, the graphene is found to be conformal over the brain surface due to its flexibility (Figure 6 (D)). Epilepsy in the rat was induced by injecting Penicillin G sodium, epilespyactivity related signals resulting from neuronal spikes with frequencies up to $25 \mathrm{~Hz}$ were then recorded using the graphene FETs as shown in (Figure 6 (E)). Finally, the author also demonstrate the potential of graphene FET array for multiplexed measurements. The results highlight the importance of new device designs and advanced nanomaterials to obtain neural probes with enhanced spatial resolution and performance.

In addition. In 2018, Velive F. et al. used graphene based FETs in the detection of ion activity in the channel when exposed to cultured hippocampal neuron networks [99]. Graphene is grown using chemical vapor depsition and resulted in stable measured ion currents in FETs with different channel sizes [100-107]. Moreover, Velive F. et al. focused the role of grain boundaries (Figures 7 (A-B)) in 

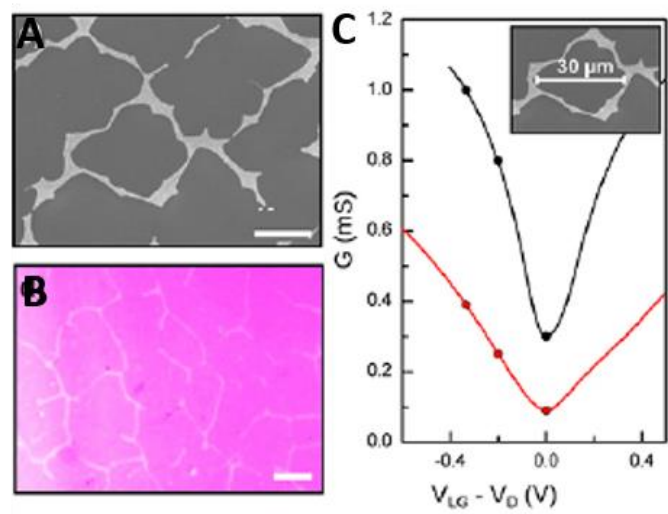

Figure 7. (A) Optical image and (B) scanning electron micrographs showing the grain boundary network (bright area). Scale bars $15 \mu \mathrm{m}$. (C) Global conductance due to one grain boundary estimated from the conductance of the grapheme FET without the grain boundary (black curve) and with a single grain boundary (red curve). Reprinted with permission from [99].

enhancing neural activity monitoring by transmitting electrons and improving ion trapping and diffusion in the graphene channel (Figure 7 (C)).

The main advantage of the highly crumpled graphene FET for neural activity recording is the ease of fabrication of the device compared to the other technologies, in addition to the large crumpled graphene area resulting in high sensitivity. However, in terms of resolution, the graphene FET is still disadvantageous. In fact, the gate dimensions of the FET array should be of the same order of magnitude of the biological building blocks (few nanometers) to be effective when used to stimulate and record neural signals as previously explained. However, this requires the reduction of the active graphene area which results in a lower sensitivity. This poses a fundamental challenge to enhance the spatial resoltuion of graphene-based FETs for neural probe application.

\subsection{Nanotube field effect transistors}

Recently, a novel architechture of field effect transistors based on nanotube (NT) FETs has been developed by Hussain M. M. et al [108]. With this architechture (Figure 8 (A)), the authors have shown that NT based FETs are more advantageous over nanowire FETs in terms of smaller area footprint and similar power dissipation [109-110]. The fabrication process is CMOS compatible and includes patterning the nanotube using electron beam lithography in addition to the epitaxial growth of source, drain and channel. In fact, NT based FETs allow for improved electrostatics (lower subthreshold slope), higher drive current, better immunity to short channel effects, and higher carrier mobility than nanowire FETs (Figure 8 (B)). A full volume inversion is demonstrated when the NT thickness is below

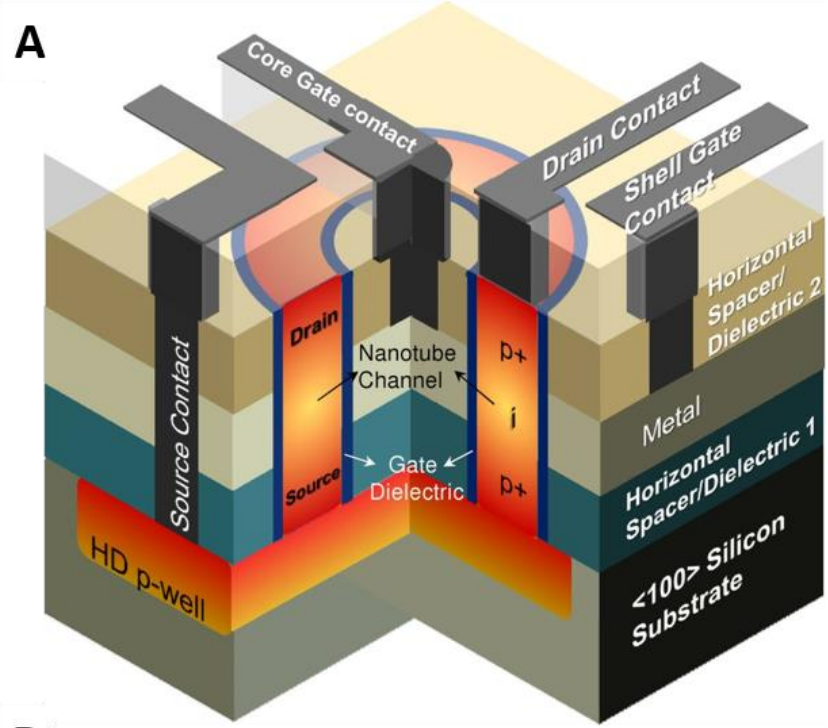

B

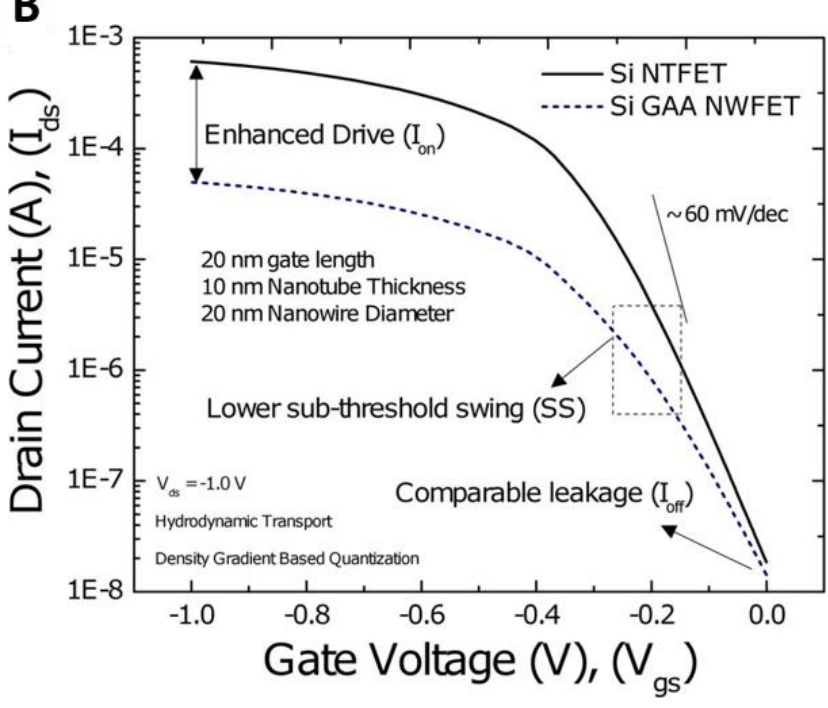

Figure 8. (A) Schematic illustration of the nanotube based field effect transistor. (B) Id-Vg curve indicates that a single silicon nanotube FET with $10 \mathrm{~nm}$ thick nanotube can provide a drive current that is 10x higher compared to a single nanowire FET with a higher sub-threshold swing and a similar off state leakage current. Reprinted with permission from [108].

20-nm. Because of volume inversion, the minority carriers in the lower energy states are able to participate in current conduction along with the surface charges. As the NT thickness is reduced, the inversion layer charge centroid shifts away from the interfaces and peaks near the center of the NT. This will result in increased carrier mobility (near ballisticity) due to reduced scattering associated with oxide/interface trapped charges and surface roughness. Even though no actual demonstration of the nanotube based FETs in BMIs has been shown yet, however, this architecture is promising in future BMI applications due to its low power dissipation and smaller footprint area than nanowire FETs. 
The nanotube FET has therefore shown advantages over the nanowire FET in terms of improved area efficiency with the similar low leakage characteristics. However, the main disadvantage over other FET technologies for BMI application lies in the more complicated and higher cost fabrication process of the devices where a larger number of lithography masks is required. The increased number of lithography masks increases the criticality of alignment. In addition, e-beam lithography is crucial for patterning the nanotube structure. The main challenge in NTFETs is in exposing the channel to the biological building blocks for an enhanced sensitivity. However, this requires the removal of the outer gate area which would consequently affect the performance of the FET in terms of gate control over the channel.

\section{Delivery Methods}

Currently, there is a growing interest in developping seamless and minimally invasive BMIs for long term probing of neuronal activity [111-112]. Current probes which are made from rigid materials such as silicon and metals have a large mechanical mismatch with the biological tissues in terms of stiffness. This results in a low coupling between device and neuron in addition to a limited stability in the long-term recordings [46, 113-114]. In fact, it has been shown that the mechanical mismatch between probes and tissues causes a micro-mothion of the porbe and results in gliar scars and chronic immune response from the heated biological tissues [46]. Recent studies have shown that the use of flexible probes fabricated on polymer substrates help in alleviating and eliminating the otherwise damaging effect on the tissues [115]. Nevertheless, current delivery techniques of flexible probes are based on surgical procedures or attachment and release from a rigid substrate which can cause severe damage to the biological cavities. Minimally invasive and seamless transfer of nanoelectronic based probes into the biological system could substantially increase their applications in intimate interfacing with iological tissues due to their flexibility, nanowire based structure and tissue-like properties [116-119].

\subsection{Syringe injectable mesh}

In 2015, Liu et al demonstrated a new syringe-injection based delivery method of macroporous probes into bioligical tissues [117]. This technique is based on (1) loading the macroprous mesh elctronics into the syringe (2) injection of the syringe into the biological tissue, (2) release of the flexible probe, (3) withdrawal of the syringe and (4) release of the inpur/output of the mesh electronic outside the body for subsequent interface connection. To successfully inject the mesh electronics into the syringe, the mesh is designed to be ultra-flexible with a bending stiffness similar to that of neural tissues $\left(10^{4}-10^{6}\right.$ smaller than previously reported stiffness for $\mathrm{Si}$ and flexible probes). This allowed the mesh electronics to roll up and get easily loaded into the syringe needle (Figure 9 (A)).

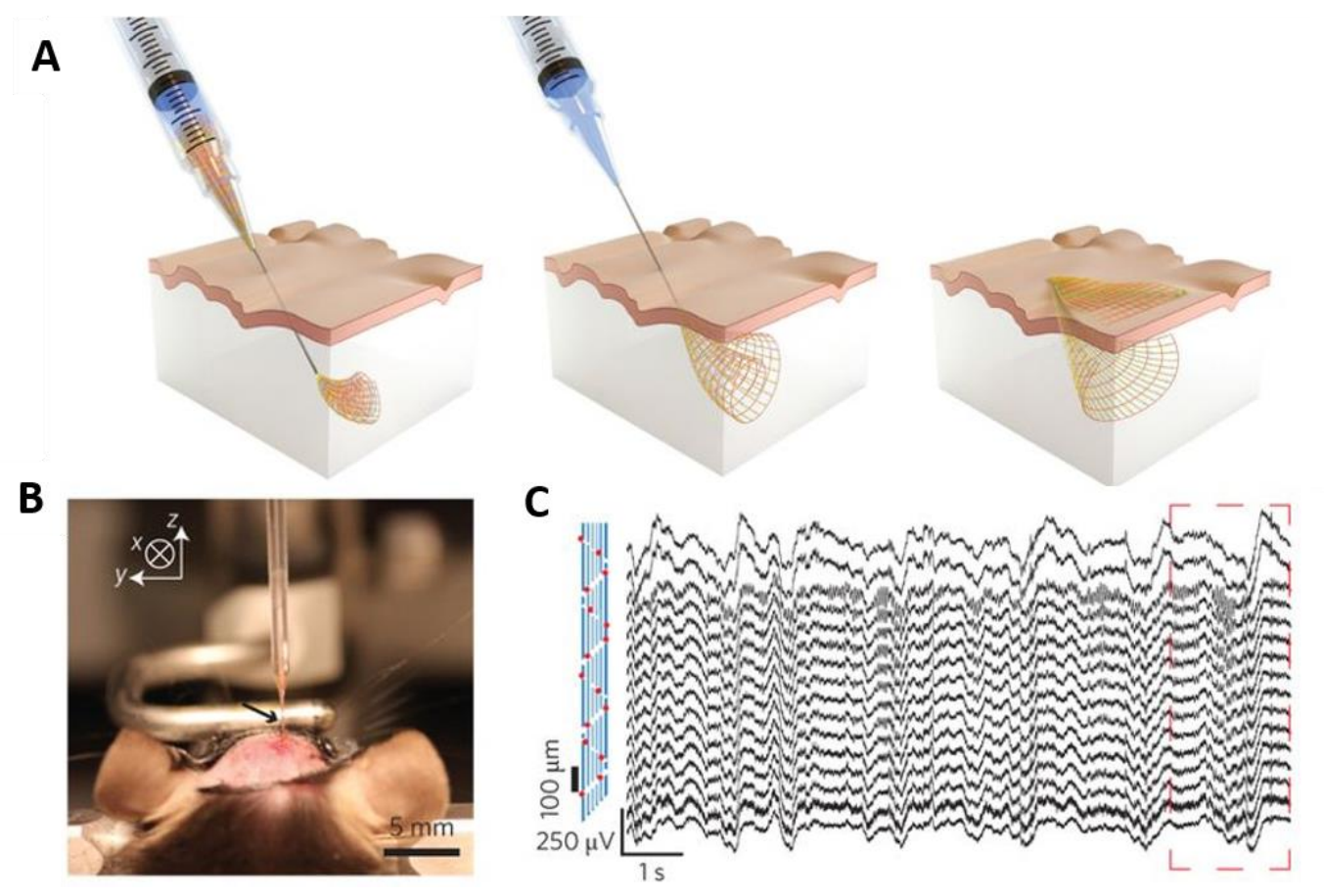

Figure 9. (A) Schematic illustration showing the injection process of the mesh electronics into biological tissues. (B) Optical image of the injection of mesh electronics into the brain of a mouse. (C) In vivo measured 16-channel electrical signals from mesh electronics injected into a mouse brain. Reprinted with permission from [117]. 
The authors confirmed the robustness and high stability of the mesh electronics before and adter injection where the injected silicon nanowre FETs showed a yield of over $90 \%$ using a needle with inner diameter of $260-600 \mu \mathrm{m}$ while the yield slightly dropped to $83 \%$ when a needle with inner diameter of $100 \mu \mathrm{m}$, is used. In addition, the conductance of the nanowire FETs changed by less than $12 \%$ ost injection. These results show the potential of this delivery technique in brain mapping applications. In fact, to prove this point, the authors used the syringe-injectable delivery of mesh electronics to study the in vivo brain activity in the hippocampus of live rodents (Figure 9 (B)).

Using confocal microscopy imaging, the mesh electronics is shown to relax from its $200 \mu \mathrm{m}$ injection diameter (Figure 9 (C)), in addition, the mesh is found to be highly bioaccepted in dense neuronal tissues. In fact, the neurophilicity of the mesh allows it to unfold and integrate within extracellular matrices forming tight connections between the mesh and the neurons. Moreover, measurements on glial fibrillary acidic protein (GFAP) fluorescence showed no developed glial scars but healthy neurons surrounding the mesh. Finally, Liu et al. recorded the mice brain activity using the injected mesh electronics which showed an amplitude of $400 \mu \mathrm{V}$ and a frequency of $1-4 \mathrm{~Hz}$. The average duration of single AP spikes was found to be around $2 \mathrm{~ms}$. The presented results confirm the potential of mesh electronics in recoding brain activity as well as to deliver nanoscale flexible probes into biological systems for monitoring and stimulation.

\subsection{Macroporous ultra-flexible probe}

In a work by Lieber et al. in 2015, a new injection technique was developed to deliver a flexible probe into biological tissues [118]. The technique is based on freezing a flexible probe before insertion into the brain. The probe then recovers its flexibility after imlpantation. Silicon nanowires based FET have been integrated on the 3D macroporous neural probes fabricated using two-dimensioal lithography with a sacrificial layer that is later etched to release the standing probe. The probes area designed based on a mesh strcuture with $80 \%$ opem area and feature sizes below $10 \mu \mathrm{m}$ to promote interpenetration of neurons with the electronic FETs (Figures 10 (A-B)).

Moreover, the neural probe is ultra-flexible and has an effective bending stiffness similar to that of brain tissues $\left(<0.64 \times 10^{15} \mathrm{~N} . \mathrm{m}^{2}\right)$ to avoid scarring and which is several orders of magnitude smaller than that of silicon and carbon fibre. This stiffness is expected to result in mechanical interactions between the probe and tissues in the range of cellular forces (10 $\mathrm{nN}$ forces). After freezing the flexible probe in liquid nitrogen, the probe was inserted and implanted in a rodent's brain with minimal tissue Damage (Figure $10(\mathrm{~B}))$. The cylindrical geometry of the probe enables the distribution of the nanowire based FET sensors around the probe and enable multiplexed recordings (Figures 10 (C-H). In fact, the authors showed simultaneous and reliable recordings from 13 nanowire FETs (Figures 10 (I-J)) which consist of more than $80 \%$ of the sensors yield. The sensors showed similar performance as well in terms of the shape and amplitude of measured potentials Moreover, a high-signal-to-noise ratio of 7 has been achieved which confirms the close procimity between the firing neurons and sensors. The probe is also proven to be bio-acceptable. In fact, upon implantation of the probe, the brain slice shows a void within the interior of the cylinder suggesting that tissues were displaced during the implantation process. Howver, optical images of the brain slides 5-weeks post-implantation show no void which suggests that tissues interpenetrate through the macroporous probe in time to fill the void. The sensors achieve bend-out geometry as well which enable the recording from sites away from any residual tissue damage due to the implantation process. Finally, input/output connections are made between the probe and measurements electronics by bonding the remaining portion of the substrate to a printed circuit board.

Even though it is crucial in future studies to further improve these probes by increasing the number of sensors for multiplexed detection, adding neural stimulation capabilities, and enhancing chronic histology to shorter and longer times, however, the syringe injectable mesh electronics and macroporous probes show great potential in minimally invasive and seamless transfer of nanoelectronic based sensors into the biological system.

In summary, both delivery techniques are based on a macroporous flexible substrate which enables neuronal interpenetration and leads to an intimate interface between the cells and nanoelectronic devices; a feature that is required for novel electrophysiological recordings.

Compared to the macroporous ultra-flexible probe, the syringe-injectable mesh approach allows for the injection of larger flexible electronics through small injection sites (as small as the size of the syringe) to fill internal cavities when unfolded. This results in a larger area in the brain being probed by the mesh and therefore a larger spatial resolution would be achieved. On the other hand, the macroporous ultra-flexible probe can be implanted through smaller injection openings and thus results in minimal surgical and acute tissue damage. This is due to the freezing of the ultrasmall, flexible and macroporous probe and its subsequent direct implantation into the brain without the need for an external syringe.

Moreover, the macroporous probe technique allows a reliable wired transfer of recordings through the electrical input/output (I/O) ports, while in the case of the syringe injection technique, all $\mathrm{I} / \mathrm{O}$ connections for recoding signals must be introduced after injection of the mesh as it is very challenging and difficut to insert and fold the conventional 


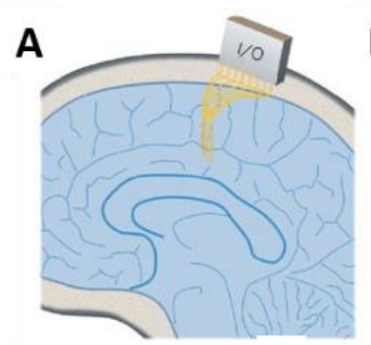

C
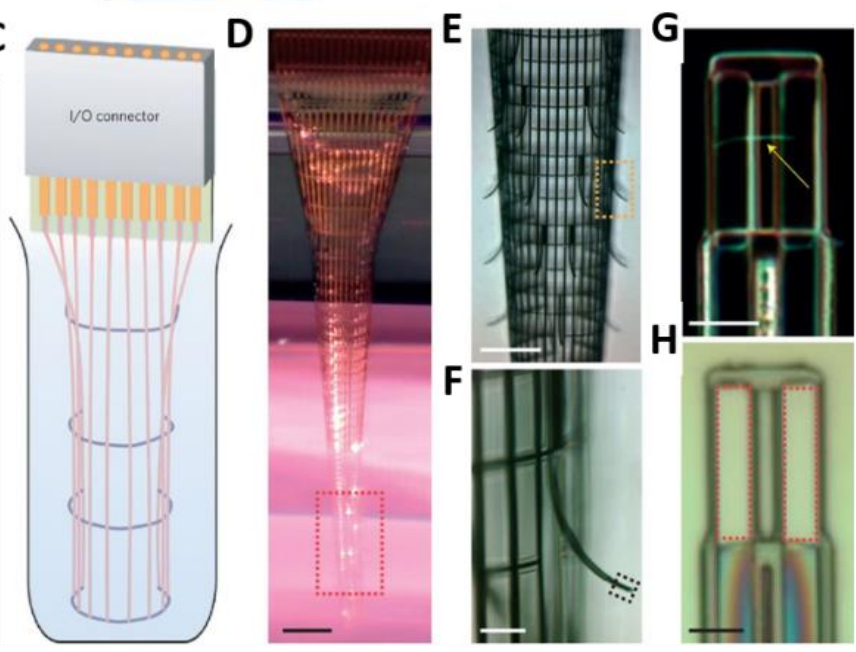
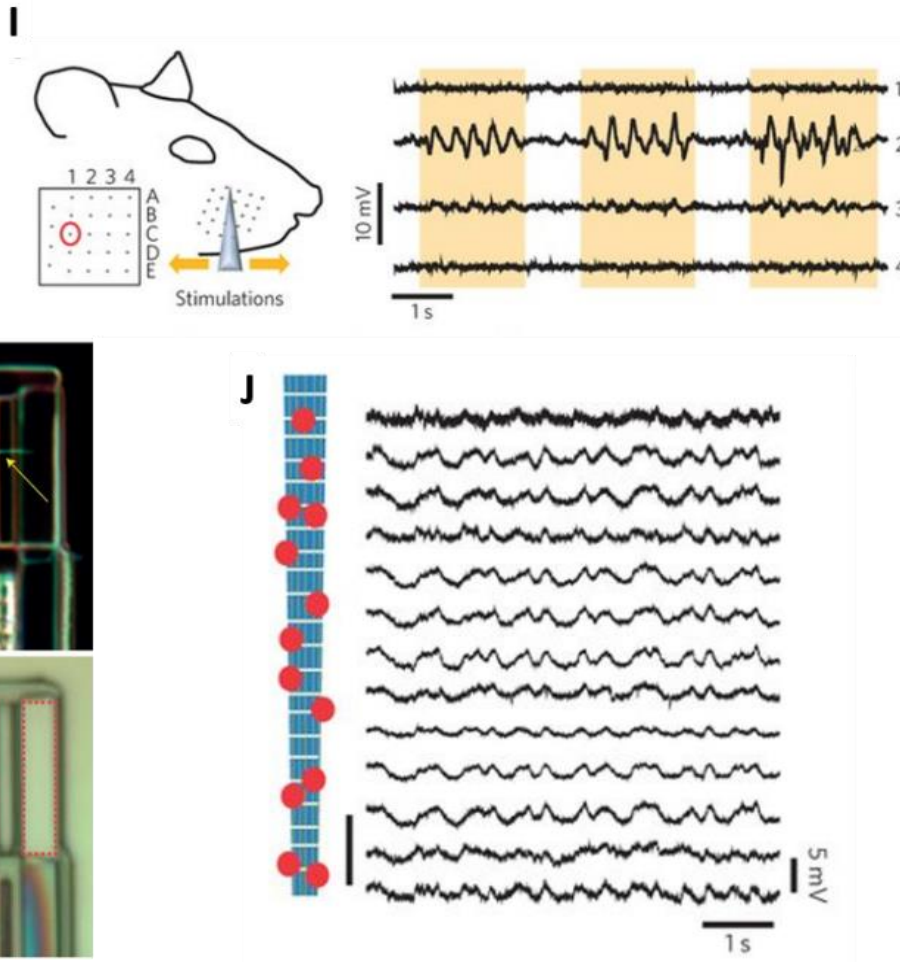

Figure 10. (A) Schematic of the implanted probe in the brain. (B) Photo of a typical rodent surgery for implantation of electronic sensors through a cranial whole. (C) Schematic of the probe with an I/O connector. (D) Optical images of the macroporous brain probe with cylindrical shape. (E) Optical image of the sensor area of the probe outlined in the red box in D. The supporting arms on the probe are outward bent. Scale bar, $200 \mu \mathrm{m}$. (F) Zoomed-in image of the outward bent supporting arm outlined in the yellow box in E. The black box depicts the sensor element. Scale bar, $50 \mu \mathrm{m}$. (G) Dark-field image of the nanowire FET voltage sensor at the end of the supporting arms. (H) Bright-field image of two platinum electrode voltage sensors. (I) Schematic of the correlation between the neural activity in the cortex and the sensory behavior of the rat (left). Schematic map of sub-areas in the barrel cortex. The red circle indicates the targeted sub-area (Inset). Traces from four neighbouring sensors with the yellow areas specifying stimulations applied to the rat (right). Positions of the four sensors are marked in the probe schematic (right). Scale bar, $200 \mu \mathrm{m}$. (J) Multiplexed signals from 13 nanowire FET sensors following probe insertion into the somatosensory cortex. Positions of the 13 sensors are marked with red dots in the schematic of the probe on the left. Scale bar, $200 \mu \mathrm{m}$ Reprinted with permission from [118].

I/O connections though the injection needles. Therefore, the main disadvantage of the syringe injectable mesh approach is that it requires the delivery of the nanoelectronics (on the mesh) and I/O connections seperately which is challenging to achieve.

Finally, it is crucial in future studies to further improve these probes by increasing the number of sensors for multiplexed detection, adding neural stimulation capabilities, and enhancing chronic histology to shorter and longer times.

\section{BMI Integrated Systems}

Even though several nanoscale transistors have been recently studied for BMI applications, however, there is still a lack in complete, tether-less, implantable BMI systems that integrate nanoscale transistor-based sensors with communication and power systems for reliable, high-resolution and long-term recordings [119-127].

\subsection{Neural dust}

The Maharbiz group studied the fundamental system design for a fully implantable and tether-less BMI system [128]. The system, called neural dust, is assessed in terms of size, power and bandwidth limits assuming it is based on low power CMOS electronics and powered using ultrasound energy (Figure $11(\mathrm{~A})$ ). A neural dust is a sub-mm wireless neural interface platform [129]. The ultra-small size of the neural dust $(10-100 \mu \mathrm{m})$ enables the massive scaling of devices for neural activity monitoring, each device having its own neural probes, power and communication system. 


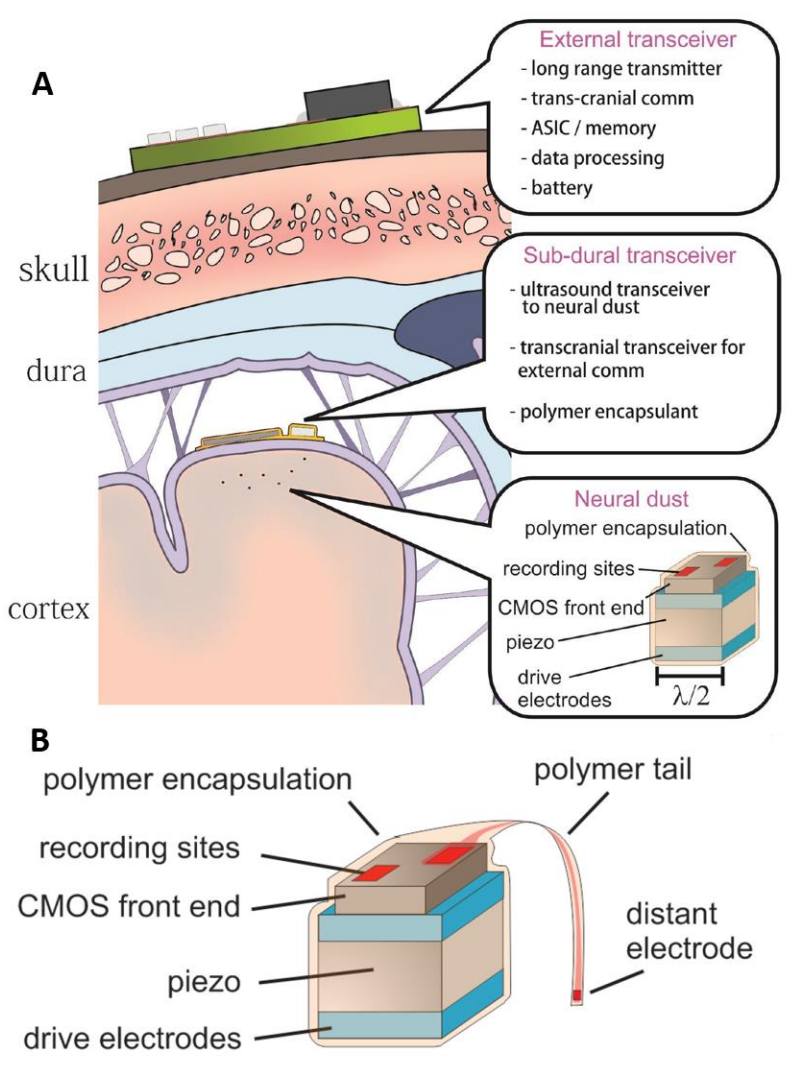

Figure 11. (A) Neural dust system depicting the placement of ultrasonic interrogator under the skull with the independent sensing nodes dispersed throughout the brain. (B) Neural dust with an ultra-flexible and fully compliant "tail" that includes electronic sensors. Reprinted with permission from [128]. Creative Commons Attribution License CC BY 4.0.

In addition, authors suggest that an interrogator would be implanted beneath the skull to avoid attenuation of the ultrasonic energy by bones and to receive the signals and recordings from all neural dusts. The interrogator would be powered using RF power transfer from an external transceiver. Therefore, during the operation, the implanted interrogator sends ultrasound energy to the sensing nodes which communicate back the recorded signals to the interrogator by modulating the phase, frequency or amplitude of the ultrasound wave.

The authors suggest two main roles for the neural dust: either as an active node that provides power for the CMOS electronics to detect, encode and transmit signals or as a passive node that optimizes the reflectivity of the dust in terms of recorded potential. In another approach, the authors discuss the addition of neural probes in a 1-5 $\mu \mathrm{m}$ small "tail" for the neural dust as shown in Figure 11 (B) and which are relatively far from the neural dust system $(>100 \mu \mathrm{m})$. This tail containing the probes can be ultra-flexible and compliant in order to avoid causing any damage to biological tissues. With such a design, the noise limit sets the power bound to $0.4 \mu \mathrm{m}$ transistors with a sensitivity of $2.3 \times 10^{-3} \mathrm{ppm}$, which corresponds to a backscattered power of $16.6 \mathrm{pW}$ at the input of the interrogator. This approach has not been tested yet, however, it provides a path towards a truly chronic implantable BMI.

In summary, the neural dust platform is a batteryless system that interfaces with the neural system using ultrasonic power and communication. Unlike other system integration approaches where an on-board bulky battery is required, the nueral dust allows the powering and data communication wirelessly using ultrasound. Previous studies have also shown that neural dust is capable of transmitting physiological data with high fidelty using the ultrasound approach. This leads to a complete chronic and low power BMI system for tetherless recordings and which could be shrank well below the mm-scale.

Nevertheless, the neural dust faces several challenges to prove effective. First, the size of the dust mote needs to be further scaled down to reduce the biological response generated due to the brain implants and cause the degradation of the recordings quality. This requires scaling down the size of the piezocrystal, however, this would affect the signal-to-noise ratio of the backscatter link and therefore reduce the sensitivity and noise performance of the dust mote. This would also affect the communication between the multiple dust motes. In addition, the added size during the packaging and assembly steps needs to be shrunk using improved microfabrication techniques with high-volume parallel fabrication.

Another challenge is the delivery technique of the dust motes into the desired cortical targets with minimal damage. The current asymmertrical design of the dust motes requires the use of coordinates to specify their orientation in the cortex, thus, better designs could be developed to facilitate the delivery and deployment of the dust motes and to enhace their stimulation capacilities.

\subsection{Out of-plane sensor electrode array integration}

One of the important most important features for any brain computer interfacing device is its biocompatibility. This basically translates to the acceptance and integration of the foreign object/device with internal biological environment without any discomfort and having any ill effects. Considering different advances in the technologies and presented examples, we can certainly say that these technologies are focused on miniaturising the devices to improve the resolution. Moreover, any electronic systems is composed of individual elements integrated either on differnet platforms or on a single platform like a printed ciruit board. The nanoscale devices individually present great perspective, however, they have to be placed on a single platform making it larger and in most of the cases rigid. Thus, theses rigid structures create a discomfort and are not 
suitable for the complex structures especially that of the internal organs [130-138].

Considering many emerging applications a new frontier has opened in last decade in electronics to achieve flexible electronics that can conform to complex structures without significant degradation in performance. Among several notable efforts in the field, Hussain et. al. has presented the most pragmatic approach for obtaining high performance flexible electronic systems by using CMOS technoology unlike other prominent names in the field who use organic devices or other nanomaterials [139]. Hussain et. al. has recently proposed a concept of out-of-plane integration strategy for flexible electronics where sensors can be integrated with the requisite electronic systems using a through polymer vias (TPV) and through silicon vias (TSV). In this research, he has shown seamless interconnection of the sensory electrodes on one side of the polymeric interface (polyimide (PI)- most widely used popular in the flexible electronics field due to its mechanical stabilities) to the connecting pads on the opposite side of interface using TPV. This process is well illustrated using the schematic and the advantage is the seamless bottom interface with the sensory electrode that does not have any protrusion even in submicron level to have any discomfort of any kind for the intended applications (Figure 12).

The interconnection using TPV is achieved by using a relatively cheap and easy to achieve method of electrochemical deposition and the electrical reliability in terms of resistance from the sensory electrodes vertically to the top electrode is studied. In addition, the approach of having the sensors electrodes on PI and then bonding it with another Si substrate having a replica of electrodes using bonding polymers like SU-8 has been achieved. The reliable interconnection of multilayer $\mathrm{Si}$ and polymer electrodes with TPV and TSV can be seen in the SEM figure. This is the first demonstration of the concept of the out-of-plane integration strategy which can be capitalized on for BMI and implantable applications.

\subsection{D-IC coin integration}

For any implantable electronic system, when very cramped and very limited space constraints are available, it is imperative to have the smallest form factor for the devices to be placed in these spaces. Thus, to fit into intracranial spaces and be able to comply with the complex hills and vallys of the most delicate organ, we must have truly flexible electronic systems that are not only capable of handling all the data processing, storage and communication functions but also should be very thin and small enough as been described in earlier sections. In addition, these devices should be packaged in biocomptaible packaging not to have any adverse effects and glial cell formations. Demonstrations throughout the research commuity in flexible or implantable
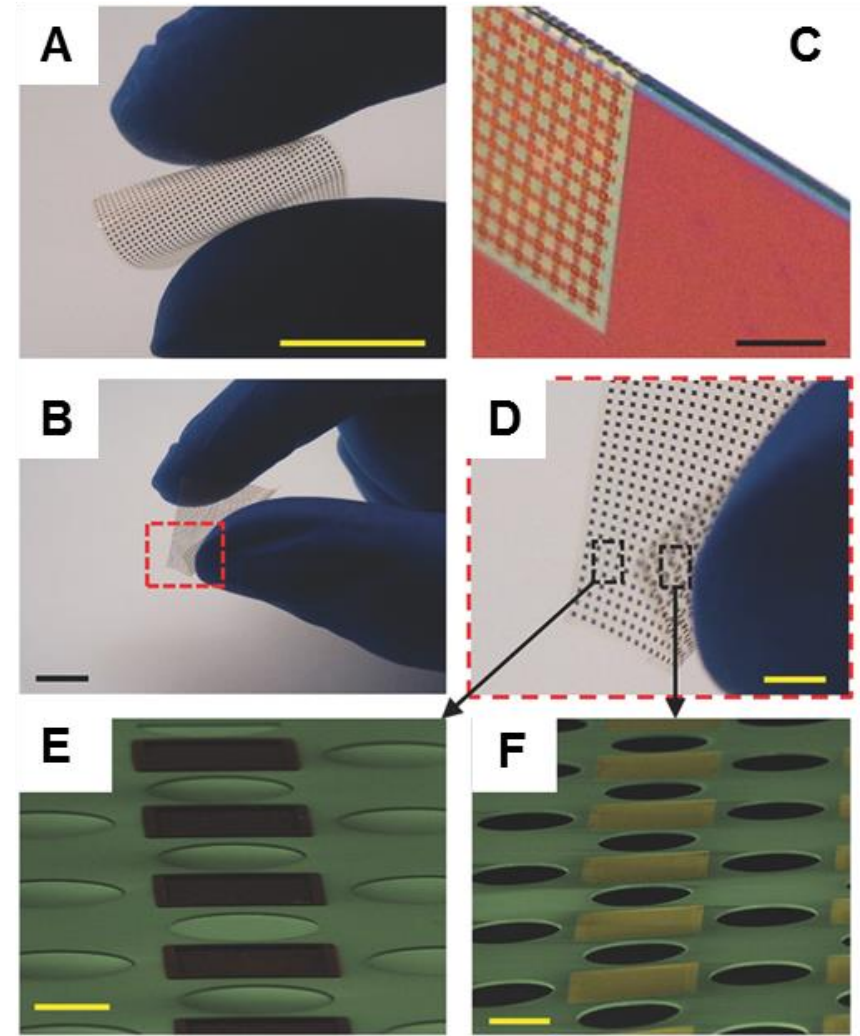

Figure 12. (A) and (B) The fabricated polyimide based electrode array can be easily handled without the use of sophisticated tools. Scale bars, $1 \mathrm{~cm}$. c) The electrode array is wrapped around an 0.5 $\mathrm{mm}$ silicon wafer with $\mathrm{SiO}_{2}$. The structure shows a bending radius of $250 \mu \mathrm{m}$. Scale bar, $1 \mathrm{~mm}$. d) Optical image of the electrode array. Scale bar, $1 \mathrm{~cm}$. Scanning electron microscopy (SEM) images of e) the top and $\mathrm{f}$ ) bottom side of the polyimide based electrode array. Scale bars, $100 \mu \mathrm{m}$. Reprinted with permission from [138].

domain focus on either making flexible sensors or demonstrating the individual flexible components, however, in reality the whole system needs to be presented and made flexible without which the concept of best BMI device with minimum invasiveness and minimum discomfort cannot be achieved.

Considering these challenges, Shaikh et al. has been working on development of a truly compliant stand-alone electronic system that can address all the issues mentioned so far in more efficient way. The challenges of integrating millions of sensors with the CMOS based high performance electronics and data storage units and the communication capabilites are addressed with their approach of 3D heterogeneous integration strategies. Planar integration of millions of sensors will occupy very large area in addition, the interconnect and the processing electronics will be directly in the vicinity of the sensing area that causes multiple issues. Shaikh et al. has presented a heterogeneous 3D integration where CMOS based devices are flexed using soft-back-etch approach, then integrated with the nanosensors that can be fabricated from nanomaterials using 
vertical TPV interconnects. The processing and data storage are CMOS based devices (unmatched with any technology out there in terms of manufacturability or the maturity, while all other technologies are still in infancy) which are not only highly reliable but also isolated from the sensing area protected by the polymer encapsulation. For communication purposes, antenna can be fabricated on the top side of the electronics unit which is also isolated and using a combination of TPV and TSV, it is interconnected. This makes an entire stand-alone system possible and area efficient for implementing millions of sensors in a very tightly packed space in a truly compliant manner. Not only the area efficiency, but also the packaging of these devices using soft-polymeric encapsulation provides the biocompatibility and the reliability for the prolonged sustained performance without degradation.

To summarize, the 3D heterogeneous and multidimensional integration strategies which are illustrated to be manufacturable using the current state-of-the-art amture CMOS based technologies promises a certain leap in the BMI applications with great pragmatism.

\section{Conclusion}

In this review, different BMI approaches based on electrical, magnetic resonance, optical and molecular based monitoring of neurons activity are first discussed with their challenges and limitations. The following sections of the review are focused on the electrical detection of neural activity with a focus on nanoscale transistors.

In the second section of the review, the design criteria for nanoscale FETs are discussed in terms of spatio-temporal resolution, power dissipation, volume displacement and biocompatibility. Different types of brain neurons fire spikes at different rates, however, a minimum sampling rate of 1 $\mathrm{kHz}$ is necessary in order to capture the simultaneous and fast spikes with a single spike resolution. It is also required that BMIs do not dissipate more than $40 \mathrm{~mW}$ and do not provide a power density above $\sim 10 \mathrm{~mW} / \mathrm{cm}^{2}$ for radio frequency electromagnetic radiation, which would otherwise cause a local rise in temperature above $2^{\circ} \mathrm{C}$ and cause biological tissue damage. In addition, the neuronal activity monitoring technique should generally not cause a volume displacement beyond $1 \%$ to avoid the disruption of the normal brain functions. The recording sensors should also be biocompatible, otherwise glial scars would be generated resulting in measurements with low signal-to-noise ratio.

In the third section of the review, we discussed nanoscale transistors used as sensors for recording and stimulating neural activity. Nanostructures present multiple advantages in biosensing applications over larger scale structures due to their high surface-to-volume ratio, resulting in improved sensitivity, and to their small size, resulting in better spatial resolution (single neuron resolution). The small size of nanostructures enables seamless integration of the FETs with biological cells as well. Representative sensing studies of neural activity were then reviewed, including stimulation and detection of multiplexed neural signals and recognition proteins released from neurons. Three key nanomaterials used as the FET sensing media are presented: silicon nanowires, carbon nanotubes and graphene sheets. These studies have clearly demonstrated the success of the nanoscale FETs in recording high rate neural spikes and in diagnosing different diseases such as Paskinson's, Alzheimer's and neuronal cancer. We then discussed several basic challenges as well as potential research directions that could further enhance the capabilities of nanoscale FET neuronal and bio-sensors, including nanotube based FETs which show better area efficiency than nanowire based FETs but with a similar leakage current. Despite advances in recent years in nanoscale FETs, improvements are still needed in terms of their ability to record and stimulate simaltaneously a larger number of neurons.

The fourth section of the review focused on different delivery techniques of the nanoscale FETs into the biological building blocks without causing any damage to the tissues and without the need for surgical operations. Syringeinjectable mesh electronics is based on (1) loading the macroprous mesh elctronics into the syringe (2) injection of the syringe into the biological tissue, (2) release of the flexible probe, (3) withdrawal of the syringe and (4) release of the inpur/output of the mesh electronic outside the body. It is essential that the mesh is ultra-flexible in order for it to be loaded into the syringe without getting damaged. Thus, the mesh is designed to have a bending stiffness similar to that of the neuronal tissues. Another promising delivery technique is based on an ultra-flexible macroporous probe which is frozen using liquid nitrogen before insertion. Once implanted, the probe recovers its flexibility. In addition, the probe is designed to have $80 \%$ open area so that tissues interpenetrate through the macroporous probe in time and interact with the integrated nanoscale FETs on the probe. Syringe-injectable mesh electronics and macroporous flexible probes show great potential in minimally invasive and seamless transfer of nanoelectronic based sensors into the biological system.

In the final section, the integration of nanoscale FETs into a complete BMI system that includes communication and power systems is explained with a focus on the neural dust concept. The neural dust technique assumes that an interrogator, powered using RF power transfer from an external transceiver, would be implanted under the skull and would provide ultrasound energy to the ultra-small sensing nodes. The sensing probes would then communicate the recorded signals back to the interrogator. Even though this approach has not been tested yet, however, it provides a path towards a truly chronic implantable BMI. Finally, we introduced an out-of-plane integration strategy for flexible 
electronics and 3D-IC coin electronics as potential integration techniques for BMI applications due to their improved fexibility, heat dissipation and area efficiency.

\section{Acknowledgements}

Authors acknowledge generous support by the King Abdullah University of Science and Technology (KAUST) Office of Sponsored Research (OSR), Award No. Sensor Innovation Initiative OSR-2015-Sensors-2707 and KAUSTKFUPM Special Initiative OSR-2016-KKI-2880.

\section{References}

[1] Alivisatos, A.P., Chun, M., Church, G.M., Greenspan, R.J., Roukes, M.L. and Yuste, R., 2012. The brain activity map project and the challenge of functional connectomics. Neuron, 74(6), pp.970-974.

[2] Bansal, A.K., Truccolo, W., Vargas-Irwin, C.E. and Donoghue, J.P., 2011. Decoding 3-D reach and grasp from hybrid signals in motor and premotor cortices: spikes, multiunit activity and local field potentials. American Journal of Physiology-Heart and Circulatory Physiology.

[3] Gerhard, F., Kispersky, T., Gutierrez, G.J., Marder, E., Kramer, M. and Eden, U., 2013. Successful reconstruction of a physiological circuit with known connectivity from spiking activity alone. PLoS computational biology, 9(7), p.e1003138.

[4] El-Atab, N and Hussain, M.M. 2018. The Future of CMOS: More Moore or a New Disruptive Technology? In Advanced Nanoelectronics: Post-Silicon Materials and Devices, Wiley, pp. 1-31.

[5] Berger, H., 1929. Über das elektrenkephalogramm des menschen. European archives of psychiatry and clinical neuroscience, 87(1), pp.527-570.

[6] Vidal, J.J., 1973. Toward direct brain-computer communication. Annual review of Biophysics and Bioengineering, 2(1), pp.157-180.

[7] Stevenson, I.H. and Kording, K.P., 2011. How advances in neural recording affect data analysis. Nature neuroscience, 14(2), p.139.

[8] Ahrens, M.B., Orger, M.B., Robson, D.N., Li, J.M. and Keller, P.J., 2013. Whole-brain functional imaging at cellular resolution using light-sheet microscopy. Nature methods, 10(5), p.413.

[9] Ziv, Y., Burns, L.D., Cocker, E.D., Hamel, E.O., Ghosh, K.K., Kitch, L.J., El Gamal, A. and Schnitzer, M.J., 2013. Long-term dynamics of CA1 hippocampal place codes. Nature neuroscience, 16(3), p.264.

[10] Kording, K.P., 2011. Of toasters and molecular ticker tapes. PLoS Computational Biology, 7(12), p.e1002291.

[11] Zamft, B.M., Marblestone, A.H., Kording, K., Schmidt, D., Martin-Alarcon, D., Tyo, K., Boyden, E.S. and Church, G., 2012. Measuring cation dependent DNA polymerase fidelity landscapes by deep sequencing. PloS one, 7(8), p.e43876.

[12] Glaser, J.I., Zamft, B.M., Marblestone, A.H., Moffitt, J.R., Tyo, K., Boyden, E.S., Church, G. and Kording, K.P., 2013. Statistical analysis of molecular signal recording. PLoS computational biology, 9(7), p.e1003145.
[13] Kandel, E.R., Markram, H., Matthews, P.M., Yuste, R. and Koch, C., 2013. Neuroscience thinks big (and collaboratively). Nature Reviews Neuroscience, 14(9), p.659.

[14] Merriam, S.M.E., Srivannavit, O., Gulari, M.N. and Wise, K.D., 2011. A three-dimensional 64-site folded electrode array using planar fabrication. Journal of Microelectromechanical Systems, 20(3), pp.594-600.

[15] Zhang, A. and Lieber, C.M., 2015. Nano-

bioelectronics. Chemical reviews, 116(1), pp.215-257.

[16] Bergveld, P., 1972. Development, operation, and application of the ion-sensitive field-effect transistor as a tool for electrophysiology. IEEE Transactions on Biomedical Engineering, (5), pp.342-351.

[17] Patolsky, F., Timko, B.P., Yu, G., Fang, Y., Greytak, A.B., Zheng, G. and Lieber, C.M., 2006. Detection, stimulation, and inhibition of neuronal signals with high-density nanowire transistor arrays. Science, 313(5790), pp.1100-1104.

[18] Geim, A.K., 2009. Graphene: status and prospects. science, 324(5934), pp.1530-1534.

[19] Zhang, Y., Tan, Y.W., Stormer, H.L. and Kim, P., 2005. Experimental observation of the quantum Hall effect and Berry's phase in graphene. nature, 438(7065), p.201.

[20] Kim, K.S., Zhao, Y., Jang, H., Lee, S.Y., Kim, J.M., Kim, K.S., Ahn, J.H., Kim, P., Choi, J.Y. and Hong, B.H., 2009. Large-scale pattern growth of graphene films for stretchable transparent electrodes. nature, 457(7230), p.706.

[21] Cohen-Karni, T., Qing, Q., Li, Q., Fang, Y. and Lieber, C.M., 2010. Graphene and nanowire transistors for cellular interfaces and electrical recording. Nano letters, 10(3), pp.10981102.

[22] Hess, L.H., Jansen, M., Maybeck, V., Hauf, M.V., Seifert, M., Stutzmann, M., Sharp, I.D., Offenhäusser, A. and Garrido, J.A., 2011. Graphene transistor arrays for recording action potentials from electrogenic cells. Advanced Materials, 23(43), pp.5045-5049.

[23] Chadderton, P., Margrie, T.W. and Häusser, M., 2004. Integration of quanta in cerebellar granule cells during sensory processing. Nature, 428(6985), p.856.

[24] Gittis, A.H., Moghadam, S.H. and Du Lac, S., 2010. Mechanisms of sustained high firing rates in two classes of vestibular nucleus neurons: differential contributions of resurgent Na, Kv3, and BK currents. American Journal of Physiology-Heart and Circulatory Physiology.

[25] Harris, J.J., Jolivet, R. and Attwell, D., 2012. Synaptic energy use and supply. Neuron, 75(5), pp.762-777.

[26] Tavakoli, M., Turicchia, L. and Sarpeshkar, R., 2010. An ultra-low-power pulse oximeter implemented with an energyefficient transimpedance amplifier. IEEE transactions on biomedical circuits and systems, 4(1), pp.27-38.

[27] Lennie, P., 2003. The cost of cortical computation. Current biology, 13(6), pp.493-497.

[28] Shoham, S., O'Connor, D.H. and Segev, R., 2006. How silent is the brain: is there a "dark matter" problem in neuroscience?. Journal of Comparative Physiology A, 192(8), pp.777-784.

[29] Barth, A.L. and Poulet, J.F., 2012. Experimental evidence for sparse firing in the neocortex. Trends in neurosciences, 35(6), pp.345-355. 
[30] Markram, H., Gerstner, W. and Sjöström, P.J., 2011. A history of spike-timing-dependent plasticity. Frontiers in synaptic neuroscience, 3 , p.4.

[31] Babadi, B. and Abbott, L.F., 2013. Pairwise analysis can account for network structures arising from spike-timing dependent plasticity. PLoS computational biology, 9(2), p.e1002906.

[32] Gire, D.H., Restrepo, D., Sejnowski, T.J., Greer, C., De Carlos, J.A. and Lopez-Mascaraque, L., 2013. Temporal processing in the olfactory system: can we see a smell?. Neuron, 78(3), pp.416-432.

[33] Taillefumier, T. and Magnasco, M.O., 2013. A phase transition in the first passage of a brownian process through a fluctuating boundary with implications for neural coding. Proceedings of the National Academy of Sciences, 110(16), pp.E1438-E1443.

[34] Schneidman, E., Berry II, M.J., Segev, R. and Bialek, W., 2006. Weak pairwise correlations imply strongly correlated network states in a neural population. Nature, 440(7087), p.1007.

[35] Maguire, Y.G., Shapiro, M.G., Cybulski, T.R., Glaser, J.I., Amodei, D., Stranges, P.B., Kalhor, R., Dalrymple, D.A., Seo, D., Alon, E. and Maharbiz, M.M., 2013. Physical principles for scalable neural recording. Frontiers in computational neuroscience, 7, p.137.

[36] Wolf, P.D. and Reichert, W.M., 2008. Thermal considerations for the design of an implanted cortical brainmachine interface (BMI). Indwelling Neural Implants: Strategies for Contending with the In Vivo Environment, pp.3338.

[37] Smith, K.D. and Zhu, L., 2010. Brain hypothermia induced by cold spinal fluid using a torso cooling pad: theoretical analyses. Medical \& biological engineering \& computing, 48(8), pp.783-791.

[38] Sukstanskii, A.L. and Yablonskiy, D.A., 2004. An analytical model of temperature regulation in human head. Journal of thermal biology, 29(7-8), pp.583-587.

[39] Lazzi, G., 2005. Thermal effects of bioimplants. IEEE Engineering in Medicine and Biology Magazine, 24(5), pp.7581.

[40] Sukstanskii, A.L. and Yablonskiy, D.A., 2006. Theoretical model of temperature regulation in the brain during changes in functional activity. Proceedings of the National Academy of Sciences, 103(32), pp.12144-12149.

[41] IEEE. (2006). C95.1-2005. IEEE Standard for Safety Levels with Respect to Human Exposure to Radio Frequency Electromagnetic Fields, 3 khzto300 ghz. Available online at: http://ieeexplore.ieee.org/xpl/login. $j s p ? t p=$ \&arnumber $=1626482$

[42] IEC. 2007. IEC60825:Safety of laser products,"in Equipment Classification and Requirements, 2nd Edn., (Geneva:International Electrotechnical Commission),6.

[43] Ribeiro, S., Gervasoni, D., Soares, E.S., Zhou, Y., Lin, S.C., Pantoja, J., Lavine, M. and Nicolelis, M.A., 2004. Longlasting novelty-induced neuronal reverberation during slowwave sleep in multiple forebrain areas. PLoS biology, 2(1), p.e24.

[44] Nicolelis, M.A., Dimitrov, D., Carmena, J.M., Crist, R., Lehew, G., Kralik, J.D. and Wise, S.P., 2003. Chronic, multisite, multielectrode recordings in macaque monkeys. Proceedings of the National Academy of Sciences, 100(19), pp.11041-11046.

[45] Blinder, P., Tsai, P.S., Kaufhold, J.P., Knutsen, P.M., Suhl, H. and Kleinfeld, D., 2013. The cortical angiome: an interconnected vascular network with noncolumnar patterns of blood flow. Nature neuroscience, 16(7), p.889.

[46] Polikov, V.S., Tresco, P.A. and Reichert, W.M., 2005. Response of brain tissue to chronically implanted neural electrodes. Journal of neuroscience methods, 148(1), pp.1-18.

[47] Ward, M.P., Rajdev, P., Ellison, C. and Irazoqui, P.P., 2009. Toward a comparison of microelectrodes for acute and chronic recordings. Brain research, 1282, pp.183-200.

[48] Prasad, A., Xue, Q.S., Dieme, R., Sankar, V., Mayrand, R., Nishida, T., Streit, W.J. and Sanchez, J.C., 2014. Abioticbiotic characterization of $\mathrm{Pt} / \mathrm{Ir}$ microelectrode arrays in chronic implants. Frontiers in neuroengineering, 7, p.2.

[49] Silver, J. and Miller, J.H., 2004. Regeneration beyond the glial scar. Nature reviews neuroscience, 5(2), p.146.

[50] Silva, G.A., 2006. Neuroscience nanotechnology: progress, opportunities and challenges. Nature reviews neuroscience, 7(1), p.65.

[51] Kotov, N.A., Winter, J.O., Clements, I.P., Jan, E., Timko, B.P., Campidelli, S., Pathak, S., Mazzatenta, A., Lieber, C.M., Prato, M. and Bellamkonda, R.V., 2009. Nanomaterials for neural interfaces. Advanced Materials, 21(40), pp.3970-4004.

[52] Alivisatos, A.P., Andrews, A.M., Boyden, E.S., Chun, M., Church, G.M., Deisseroth, K., Donoghue, J.P., Fraser, S.E., Lippincott-Schwartz, J., Looger, L.L. and Masmanidis, S., 2013. Nanotools for neuroscience and brain activity mapping.

[53] Angle, M.R., Cui, B. and Melosh, N.A., 2015. Nanotechnology and neurophysiology. Current opinion in neurobiology, 32, pp.132-140.

[54] Patolsky, F., Timko, B.P., Zheng, G. and Lieber, C.M., 2007. Nanowire-based nanoelectronic devices in the life sciences. MRS bulletin, 32(2), pp.142-149.

[55] Cui, Y., Wei, Q., Park, H. and Lieber, C.M., 2001. Nanowire nanosensors for highly sensitive and selective detection of biological and chemical species. Science, 293(5533), pp.1289-1292.

[56] Stern, E., Klemic, J.F., Routenberg, D.A., Wyrembak, P.N., Turner-Evans, D.B., Hamilton, A.D., LaVan, D.A., Fahmy, T.M. and Reed, M.A., 2007. Label-free immunodetection with CMOS-compatible semiconducting nanowires. Nature, 445(7127), p.519.

[57] Zheng, G., Patolsky, F., Cui, Y., Wang, W.U. and Lieber, C.M., 2005. Multiplexed electrical detection of cancer markers with nanowire sensor arrays. Nature biotechnology, 23(10), p.1294.

[58] Qing, Q., Pal, S.K., Tian, B., Duan, X., Timko, B.P., Cohen-Karni, T., Murthy, V.N. and Lieber, C.M., 2010. Nanowire transistor arrays for mapping neural circuits in acute brain slices. Proceedings of the National Academy of Sciences, 107(5), pp.1882-1887.

[59] Tian, B., Cohen-Karni, T., Qing, Q., Duan, X., Xie, P. and Lieber, C.M., 2010. Three-dimensional, flexible nanoscale field-effect transistors as localized bioprobes. Science, 329(5993), pp.830-834. 
[60] Chen, R.J., Choi, H.C., Bangsaruntip, S., Yenilmez, E., Tang, X., Wang, Q., Chang, Y.L. and Dai, H., 2004. An investigation of the mechanisms of electronic sensing of protein adsorption on carbon nanotube devices. Journal of the American Chemical Society, 126(5), pp.1563-1568.

[61] Gruner, G., 2006. Carbon nanotube transistors for biosensing applications. Analytical and bioanalytical chemistry, 384(2), pp.322-335.

[62] So, H.M., Won, K., Kim, Y.H., Kim, B.K., Ryu, B.H., Na, P.S., Kim, H. and Lee, J.O., 2005. Single-walled carbon nanotube biosensors using aptamers as molecular recognition elements. Journal of the American Chemical Society, 127(34), pp.11906-11907.

[63] Byon, H.R. and Choi, H.C., 2006. Network single-walled carbon nanotube-field effect transistors (SWNT-FETs) with increased Schottky contact area for highly sensitive biosensor applications. Journal of the American Chemical Society, 128(7), pp.2188-2189.

[64] Star, A., Tu, E., Niemann, J., Gabriel, J.C.P., Joiner, C.S. and Valcke, C., 2006. Label-free detection of DNA hybridization using carbon nanotube network field-effect transistors. Proceedings of the National Academy of Sciences, 103(4), pp.921-926.

[65] Li, C., Curreli, M., Lin, H., Lei, B., Ishikawa, F.N., Datar, R., Cote, R.J., Thompson, M.E. and Zhou, C., 2005.

Complementary detection of prostate-specific antigen using In2O3 nanowires and carbon nanotubes. Journal of the American Chemical Society, 127(36), pp.12484-12485.

[66] Hahm, J.I. and Lieber, C.M., 2004. Direct ultrasensitive electrical detection of DNA and DNA sequence variations using nanowire nanosensors. Nano letters, 4(1), pp.51-54.

[67] Patolsky, F., Zheng, G., Hayden, O., Lakadamyali, M., Zhuang, X. and Lieber, C.M., 2004. Electrical detection of single viruses. Proceedings of the National Academy of Sciences, 101(39), pp.14017-14022.

[68] El-Atab, N. and Nayfeh, A., 2016. 1D versus 3D quantum confinement in 1-5 $\mathrm{nm} \mathrm{ZnO}$ nanoparticle agglomerations for application in charge-trapping memory devices. Nanotechnology 27 (27), p. 275205.

[69] El-Atab, N., Ulusoy, T.G., Amir, G., Suh, J., Islam, R., Okyay, A.K., Saraswat, K. and Nayfeh, A., 2017. Cubic-phase zirconia nano-island growth using atomic layer deposition and application in low-power charge-trapping nonvolatile-memory devices. Nanotechnology 28 (44), p. 445201.

[70] El-Atab, N., Chowdhury, F., Ulusoy, T.G., Amir, G., Nazirzadeh, A., Okyay, A.K. and Nayfeh, A., 2016. 3-nm $\mathrm{ZnO}$ nanoislands deposition and application in charge trapping memory grown by single ALD step. Scientific reports 6, p. 38712.

[71] Hussain, M. and El-Atab, N. 2018. 2D materials show brain-like learning. Nature Electronics 1 (8), p. 436.

[72] Hu, H., Ni, Y., Montana, V., Haddon, R.C. and Parpura, V., 2004. Chemically functionalized carbon nanotubes as substrates for neuronal growth. Nano letters, 4(3), pp.507-511.

[73] Metz-Boutigue, M.H., Goumon, Y., Strub, J.M., Lugardon, K. and Aunis, D., 2003. Antimicrobial Chromogranins and Proenkephalin-A-Derived Peptides: Antibacterial and Antifungal Activities of Chromogranins and
Proenkephalin-A-Derived Peptides. Annals of the New York Academy of Sciences, 992(1), pp.168-178.

[74] Lechner, T., Adlassnig, C., Humpel, C., Kaufmann, W.A., Maier, H., Reinstadler-Kramer, K., Hinterhölzl, J., Mahata, S.K., Jellinger, K.A. and Marksteiner, J., 2004. Chromogranin peptides in Alzheimer's disease. Experimental gerontology, 39(1), pp.101-113.

[75] Yoo, S.H., 1994. pH-dependent interaction of chromogranin A with integral membrane proteins of secretory vesicle including 260-kDa protein reactive to inositol 1, 4, 5triphosphate receptor antibody. Journal of Biological Chemistry, 269(16), pp.12001-12006.

[76] Ciesielski-Treska, J., Ulrich, G., Taupenot, L., ChasserotGolaz, S., Corti, A., Aunis, D. and Bader, M.F., 1998. Chromogranin A induces a neurotoxic phenotype in brain microglial cells. Journal of Biological Chemistry, 273(23), pp.14339-14346.

[77] Taupenot, L., Harper, K.L. and O'connor, D.T., 2003. The chromogranin-secretogranin family. New England Journal of Medicine, 348(12), pp.1134-1149.

[78] Wang, C.W., Pan, C.Y., Wu, H.C., Shih, P.Y., Tsai, C.C., Liao, K.T., Lu, L.L., Hsieh, W.H., Chen, C.D. and Chen, Y.T., 2007. In Situ Detection of Chromogranin A Released from Living Neurons with a Single-Walled Carbon-Nanotube FieldEffect Transistor. small, 3(8), pp.1350-1355.

[79] Bernini, G.P., Moretti, A., Ferdeghini, M., Ricci, S., Letizia, C., D'Erasmo, E., Argenio, G.F. and Salvetti, A., 2001. A new human chromogranin 'A'immunoradiometric assay for the diagnosis of neuroendocrine tumours. British journal of cancer, 84(5), p.636.

[80] Geim, A.K., 2009. Graphene: status and prospects. science, 324(5934), pp.1530-1534.

[81] Zhang, Y., Tan, Y.W., Stormer, H.L. and Kim, P., 2005. Experimental observation of the quantum Hall effect and Berry's phase in graphene. nature, 438(7065), p.201.

[82] Kim, K.S., Zhao, Y., Jang, H., Lee, S.Y., Kim, J.M., Kim, K.S., Ahn, J.H., Kim, P., Choi, J.Y. and Hong, B.H., 2009. Large-scale pattern growth of graphene films for stretchable transparent electrodes. nature, 457(7230), p.706.

[83] Cohen-Karni, T., Qing, Q., Li, Q., Fang, Y. and Lieber, C.M., 2010. Graphene and nanowire transistors for cellular interfaces and electrical recording. Nano letters, 10(3), pp.10981102.

[84] Hess, L.H., Jansen, M., Maybeck, V., Hauf, M.V., Seifert, M., Stutzmann, M., Sharp, I.D., Offenhäusser, A. and Garrido, J.A., 2011. Graphene transistor arrays for recording action potentials from electrogenic cells. Advanced Materials, 23(43), pp.5045-5049.

[85] Liu, X., Demosthenous, A. and Donaldson, N., 2008. Platinum electrode noise in the ENG spectrum. Medical \& biological engineering \& computing, 46(10), pp.997-1003.

[86] Cheng, Z., Hou, J., Zhou, Q., Li, T., Li, H., Yang, L., Jiang, K., Wang, C., Li, Y. and Fang, Y., 2013. Sensitivity limits and scaling of bioelectronic graphene transducers. Nano letters, 13(6), pp.2902-2907.

[87] Jakobson, C.G. and Nemirovsky, Y., 1999. 1/f noise in ion sensitive field effect transistors from subthreshold to saturation. IEEE Transactions on Electron Devices, 46(1), pp.259-261. 
[88] Robinson, D.A., 1968. The electrical properties of metal microelectrodes. Proceedings of the IEEE, 56(6), pp.1065-1071.

[89] Malleo, D., Nevill, J.T., Van Ooyen, A., Schnakenberg, U., Lee, L.P. and Morgan, H., 2010. Note: Characterization of electrode materials for dielectric spectroscopy. Review of Scientific Instruments, 81(1), p.016104.

[90] Yang, L., Zhao, Y., Xu, W., Shi, E., Wei, W., Li, X., Cao, A., Cao, Y. and Fang, Y., 2016. Highly crumpled all-carbon transistors for brain activity recording. Nano letters, 17(1), pp.71-77.

[91] Liu, X.H., Suo, Z., Ma, Q. and Fujimoto, H., 2000. Developing design rules to avert cracking and debonding in integrated circuit structures. Engineering Fracture Mechanics, 66(4), pp.387-402.

[92] Cotterell, B. and Chen, Z., 2000. Buckling and cracking of thin films on compliant substrates under compression. International Journal of Fracture, 104(2), pp.169179.

[93] Kim, D.H., Lu, N., Ma, R., Kim, Y.S., Kim, R.H., Wang, S., Wu, J., Won, S.M., Tao, H., Islam, A. and Yu, K.J., 2011. Epidermal electronics. science, 333(6044), pp.838-843.

[94] Zhao, H., Min, K. and Aluru, N.R., 2009. Size and chirality dependent elastic properties of graphene nanoribbons under uniaxial tension. Nano letters, 9(8), pp.3012-3015.

[95] Zang, J., Ryu, S., Pugno, N., Wang, Q., Tu, Q., Buehler, M.J. and Zhao, X., 2013. Multifunctionality and control of the crumpling and unfolding of large-area graphene. Nature materials, 12(4), p.321.

[96] Leem, J., Wang, M.C., Kang, P. and Nam, S., 2015. Mechanically self-assembled, three-dimensional graphene-gold hybrid nanostructures for advanced nanoplasmonic sensors. Nano letters, 15(11), pp.7684-7690.

[97] Lacour, S.P., Wagner, S., Huang, Z. and Suo, Z., 2003. Stretchable gold conductors on elastomeric substrates. Applied physics letters, 82(15), pp.2404-2406.

[98] Zhang, M., Fang, S., Zakhidov, A.A., Lee, S.B., Aliev, A.E., Williams, C.D., Atkinson, K.R. and Baughman, R.H., 2005. Strong, transparent, multifunctional, carbon nanotube sheets. Science, 309(5738), pp.1215-1219.

[99] Veliev, F., Cresti, A., Kalita, D., Bourrier, A., Belloir, T., Briançon-Marjollet, A., Albrieux, M., Roche, S., Bouchiat, V. and Delacour, C., 2018. Sensing ion channel in neuron networks with graphene field effect transistors. 2D Materials, 5(4), p.045020.

[100] Bendali, A., Hess, L.H., Seifert, M., Forster, V., Stephan, A.F., Garrido, J.A. and Picaud, S., 2013. Purified Neurons can Survive on Peptide-Free Graphene Layers. Advanced healthcare materials, 2(7), pp.929-933.

[101] Lorenzoni, M., Brandi, F., Dante, S., Giugni, A. and Torre, B., 2013. Simple and effective graphene laser processing for neuron patterning application. Scientific reports, 3, p.1954.

[102] Park, J.J., Hyun, W.J., Mun, S.C., Park, Y.T. and Park, O.O., 2015. Highly stretchable and wearable graphene strain sensors with controllable sensitivity for human motion monitoring. ACS applied materials \& interfaces, 7(11), pp.6317-6324.

[103] Tang, M., Song, Q., Li, N., Jiang, Z., Huang, R. and Cheng, G., 2013. Enhancement of electrical signaling in neural networks on graphene films. Biomaterials, 34(27), pp.64026411 .

[104] Blaschke, B.M., Tort-Colet, N., Guimerà-Brunet, A., Weinert, J., Rousseau, L., Heimann, A., Drieschner, S., Kempski, O., Villa, R., Sanchez-Vives, M.V. and Garrido, J.A., 2017. Mapping brain activity with flexible graphene microtransistors. 2D Materials, 4(2), p.025040.

[105] Hébert, C., Masvidal-Codina, E., Suarez-Perez, A., Calia, A.B., Piret, G., Garcia-Cortadella, R., Illa, X., Del Corro Garcia, E., De la Cruz Sanchez, J.M., Casals, D.V. and PratsAlfonso, E., 2018. Flexible graphene solution-gated field-effect transistors: efficient transducers for microelectrocorticography. Advanced Functional Materials, 28(12), p. 1703976 .

[106] Kireev, D., Brambach, M., Seyock, S., Maybeck, V., Fu, W., Wolfrum, B. and Offenhäusser, A., 2017. Graphene transistors for interfacing with cells: Towards a deeper understanding of liquid gating and sensitivity. Scientific reports, 7(1), p.6658.

[107] Wang, Y.Y., Pham, T.D., Zand, K., Li, J. and Burke, P.J., 2014. Charging the quantum capacitance of graphene with a single biological ion channel. Acs Nano, 8(5), pp.4228-4238.

[108] Fahad, H.M. and Hussain, M.M., 2012. Are nanotube architectures more advantageous than nanowire architectures for field effect transistors?. Scientific reports, 2, p.475.

[109] Melosh, N.A., Boukai, A., Diana, F., Gerardot, B., Badolato, A., Petroff, P.M. and Heath, J.R., 2003. Ultrahighdensity nanowire lattices and circuits. Science, 300(5616), pp.112-115.

[110] Beckman, R., Johnston-Halperin, E., Luo, Y., Green, J.E. and Heath, J.R., 2005. Bridging dimensions: Demultiplexing ultrahigh-density nanowire circuits. Science, 310(5747), pp.465468.

[111] Alivisatos, A.P., Chun, M., Church, G.M., Deisseroth, K., Donoghue, J.P., Greenspan, R.J., McEuen, P.L., Roukes, M.L., Sejnowski, T.J., Weiss, P.S. and Yuste, R., 2013. The brain activity map. Science, 339(6125), pp.1284-1285.

[112] Birmingham, K., Gradinaru, V., Anikeeva, P., Grill, W.M., Pikov, V., McLaughlin, B., Pasricha, P., Weber, D., Ludwig, K. and Famm, K., 2014. Bioelectronic medicines: a research roadmap. Nature Reviews Drug Discovery, 13(6), p.399.

[113] Seymour, J.P. and Kipke, D.R., 2007. Neural probe design for reduced tissue encapsulation in CNS. Biomaterials, 28(25), pp.3594-3607.

[114] HajjHassan, M., Chodavarapu, V. and Musallam, S., 2008. NeuroMEMS: neural probe microtechnologies. Sensors, 8(10), pp.6704-6726.

[115] Kozai, T.D.Y. and Kipke, D.R., 2009. Insertion shuttle with carboxyl terminated self-assembled monolayer coatings for implanting flexible polymer neural probes in the brain. Journal of neuroscience methods, 184(2), pp.199-205.

[116] Hong, G., Fu, T.M., Zhou, T., Schuhmann, T.G., Huang, J. and Lieber, C.M., 2015. Syringe injectable electronics: Precise targeted delivery with quantitative input/output connectivity. Nano letters, 15(10), pp.6979-6984.

[117] Liu, J., 2018. Syringe injectable electronics. In Biomimetics Through Nanoelectronics (pp. 65-93). Springer, Cham. 
[118] Xie, C., Liu, J., Fu, T.M., Dai, X., Zhou, W. and Lieber, C.M., 2015. Three-dimensional macroporous nanoelectronic networks as minimally invasive brain probes. Nature materials, 14(12), p.1286.

[119] Biederman, W., Yeager, D.J., Narevsky, N., Koralek, A.C., Carmena, J.M., Alon, E. and Rabaey, J.M., 2013. A FullyIntegrated, Miniaturized $\left(0.125 \mathrm{~mm}^{2}\right) 10.5 \mu \mathrm{W}$ Wireless Neural Sensor. IEEE Journal of Solid-State Circuits, 48(4), pp.960970.

[120] Fan, D., Rich, D., Holtzman, T., Ruther, P., Dalley, J.W., Lopez, A., Rossi, M.A., Barter, J.W., Salas-Meza, D., Herwik, S. and Holzhammer, T., 2011. A wireless multi-channel recording system for freely behaving mice and rats. PloS one, 6(7), p.e22033.

[121] Miranda, H., Gilja, V., Chestek, C.A., Shenoy, K.V. and Meng, T.H., 2010. HermesD: A high-rate long-range wireless transmission system for simultaneous multichannel neural recording applications. IEEE Transactions on Biomedical Circuits and Systems, 4(3), pp.181-191.

[122] Szuts, T.A., Fadeyev, V., Kachiguine, S., Sher, A., Grivich, M.V., Agrochão, M., Hottowy, P., Dabrowski, W., Lubenov, E.V., Siapas, A.G. and Uchida, N., 2011. A wireless multi-channel neural amplifier for freely moving animals. Nature neuroscience, 14(2), p.263.

[123] Harrison, R.R., Watkins, P.T., Kier, R.J., Black, D.J., Lovejoy, R.O., Normann, R.A. and Solzbacher, F., 2007, January. Design and testing of an integrated circuit for multielectrode neural recording. In 20th International Conference on VLSI Design held jointly with 6th International Conference on Embedded Systems (VLSID'07)(pp. 907-912). IEEE.

[124] Ganguly, K. and Carmena, J.M., 2009. Emergence of a stable cortical map for neuroprosthetic control. PLoS biology, 7(7), p.e1000153.

[125] Turner, J.N., Shain, W., Szarowski, D.H., Andersen, M., Martins, S., Isaacson, M. and Craighead, H., 1999. Cerebral astrocyte response to micromachined silicon implants. Experimental neurology, 156(1), pp.33-49.

[126] Chestek, C.A., Gilja, V., Nuyujukian, P., Foster, J.D., Fan, J.M., Kaufman, M.T., Churchland, M.M., Rivera-Alvidrez, Z., Cunningham, J.P., Ryu, S.I. and Shenoy, K.V., 2011. Longterm stability of neural prosthetic control signals from silicon cortical arrays in rhesus macaque motor cortex. Journal of neural engineering, 8(4), p.045005.

[127] Suner, S., Fellows, M.R., Vargas-Irwin, C., Nakata, G.K. and Donoghue, J.P., 2005. Reliability of signals from a chronically implanted, silicon-based electrode array in nonhuman primate primary motor cortex. IEEE transactions on neural systems and rehabilitation engineering, 13(4), pp.524541.

[128] Seo, D., Carmena, J.M., Rabaey, J.M., Alon, E. and Maharbiz, M.M., 2013. Neural dust: An ultrasonic, low power solution for chronic brain-machine interfaces. arXiv preprint arXiv:1307.2196.

[129] El-Atab, N., Faizan, S.F., Khan, S.M. and Hussain, M.M. 2019. Bi-Facial Substrates Enabled Heterogeneous MultiDimensional Integrated Circuits (MD-IC) for Internet of Things (IoT) Applications. Advanced Engineering Materials, p. 1900043.
[130] Viventi, J., Kim, D.H., Vigeland, L., Frechette, E.S., Blanco, J.A., Kim, Y.S., Avrin, A.E., Tiruvadi, V.R., Hwang, S.W., Vanleer, A.C. and Wulsin, D.F., 2011. Flexible, foldable, actively multiplexed, high-density electrode array for mapping brain activity in vivo. Nature neuroscience, 14(12), p.1599.

[131] Khodagholy, D., Gelinas, J.N., Thesen, T., Doyle, W., Devinsky, O., Malliaras, G.G. and Buzsáki, G., 2015. NeuroGrid: recording action potentials from the surface of the brain. Nature neuroscience, 18(2), p.310.

[132] Qi, D., Liu, Z., Yu, M., Liu, Y., Tang, Y., Lv, J., Li, Y., Wei, J., Liedberg, B., Yu, Z. and Chen, X., 2015. Highly stretchable gold nanobelts with sinusoidal structures for recording electrocorticograms. Advanced Materials, 27(20), pp.3145-3151.

[133] Minev, I.R., Musienko, P., Hirsch, A., Barraud, Q., Wenger, N., Moraud, E.M., Gandar, J., Capogrosso, M., Milekovic, T., Asboth, L. and Torres, R.F., 2015. Electronic dura mater for long-term multimodal neural interfaces. Science, 347(6218), pp.159-163.

[134] Yamazaki, M., Tucker, D.M., Fujimoto, A., Yamazoe, T., Okanishi, T., Yokota, T., Enoki, H. and Yamamoto, T., 2012. Comparison of dense array EEG with simultaneous intracranial EEG for interictal spike detection and localization. Epilepsy research, 98(2-3), pp.166-173.

[135] Petroff, O.A., Spencer, D.D., Goncharova, I.I. and Zaveri, H.P., 2016. A comparison of the power spectral density of scalp EEG and subjacent electrocorticograms. Clinical Neurophysiology, 127(2), pp.1108-1112.

[136] Kozma, R. and Freeman, W.J., 2016. Cognitive phase transitions in the cerebral cortex-enhancing the neuron doctrine by modeling neural fields. Switzerland: Springer International Publishing.

[137] Rojas, J.P., Torres Sevilla, G.A., Ghoneim, M.T., Inayat, S.B., Ahmed, S.M., Hussain, A.M. and Hussain, M.M., 2014. Transformational silicon electronics. ACS nano, 8(2), pp.14681474.

[138] Hussain, A.M. and Hussain, M.M., 2016. CMOStechnology-enabled flexible and stretchable electronics for internet of everything applications. Advanced Materials, 28(22), pp.4219-4249.

[139] Hussain, A.M. and Hussain, M.M., 2016. Deterministic Integration of Out-of-Plane Sensor Arrays for Flexible Electronic Applications. Small, 12(37), pp.5141-5145. 